OPEN ACCESS

Edited by:

Sergio Oscar Angel,

CONICET Instituto Tecnológico de

Chascomús (INTECH), Argentina

Reviewed by:

Alpha Fardah Athiyyah,

Airlangga University, Indonesia

Mark Poritz,

Idaho Molecular Inc., United States

Graciela Castro Escarpulli,

Instituto Politécnico Nacional de

México (IPN), Mexico

*Correspondence:

Stéphane Bonacorsi

stephane.bonacorsi@aphp.fr

Specialty section:

This article was submitted to Infectious Diseases - Surveillance,

Prevention and Treatment,

a section of the journal

Frontiers in Medicine

Received: 26 May 2021

Accepted: 19 August 2021

Published: 23 September 2021

Citation:

Bonacorsi S, Visseaux B, Bouzid D,

Pareja J, Rao SN, Manissero D,

Hansen G and Vila J (2021)

Systematic Review on the Correlation of Quantitative PCR Cycle Threshold Values of Gastrointestinal Pathogens With Patient Clinical Presentation and

Outcomes. Front. Med. 8:711809.

doi: 10.3389/fmed.2021.711809

\section{Systematic Review on the Correlation of Quantitative PCR Cycle Threshold Values of Gastrointestinal Pathogens With Patient Clinical Presentation and Outcomes}

\begin{abstract}
Stéphane Bonacorsi ${ }^{1 *}$, Benoit Visseaux ${ }^{2,3}$, Donia Bouzid ${ }^{2,4}$, Josep Pareja ${ }^{5}$, Sonia N. Rao ${ }^{6}$, Davide Manissero ${ }^{7}$, Glen Hansen ${ }^{8,9}$ and Jordi Vila ${ }^{10}$

${ }^{1}$ Department of Microbiology, Robert Debré University Hospital, AP-HP, Paris, France, ${ }^{2}$ Université de Paris, IAME, INSERM, Paris, France, ${ }^{3}$ Université de Paris, Laboratoire de Virologie, Hôpital Bichat Claude Bernard, Assistance Publique-Hôpitaux de Paris, Paris, France, ${ }^{4}$ Université de Paris, Service d'Accueil des Urgences, Hôpital Bichat Claude Bernard, Assistance Publique-Hôpitaux de Paris, Paris, France, ${ }^{5}$ STAT-Dx Life, S.L. (a QIAGEN Company), Medical Affairs, Barcelona, Spain, ${ }^{6}$ QIAGEN Inc., Medical Affairs, Germantown, MD, United States, ${ }^{7}$ QIAGEN Manchester Ltd, Medical Affairs, Manchester, United Kingdom, ${ }^{8}$ Microbiology and Molecular Diagnostics, Hennepin County Medical Center, Department of Infectious Diseases, School of Medicine, University of Minnesota, Minneapolis, MN, United States, ${ }^{9}$ Department of Pathology and Laboratory Medicine, School of Medicine, University of Minnesota, Minneapolis, MN, United States, ${ }^{10}$ Biomedical Diagnostic Centre, Department of Clinical Microbiology, Institute of Global Health, School of Medicine, Hospital Clinic, University of Barcelona, Barcelona, Spain
\end{abstract}

Background: Quantitative (q) polymerase chain reaction (PCR) cycle threshold (Ct) values represent the number of amplification cycles required for a positive PCR result and are a proxy of pathogen quantity in the tested sample. The clinical utility of Ct values remains unclear for gastrointestinal infections.

Objectives: This systematic review assesses the global medical literature for associations between Ct values of gastrointestinal pathogens and patient presentation and clinical outcomes.

Data Sources: MEDLINE, EMBASE, Cochrane library databases: searched January 14-17, 2020.

Study Eligibility Criteria: Studies reporting on the presence or absence of an association between Ct values and clinical outcomes in adult and pediatric populations were included. Animal studies, reviews, meta-analyses, and non-English language studies were excluded.

Participants: Humans infected with gastrointestinal pathogens, detected with qPCR.

Interventions: Diagnostics assessing Ct values. Extracted data were reported narratively.

Results: Thirty-three eligible studies were identified; the most commonly studied pathogens were Clostridioides difficile $(n=15)$, norovirus $(n=10)$, and rotavirus $(n=9)$. Statistically significant associations between low $C$. difficile $C t$ values and increased symptom severity or poor outcome were reported in 4/8 (50\%) studies, and increased risk of death in 1/2 (50\%) studies; no significant associations were found between Ct value 
and duration of symptoms or length of hospital stay. Among studies of norovirus, 5/7 $(71 \%)$, mainly genogroup II, reported symptomatic cases with significantly lower median Ct values than controls. Significantly lower rotavirus Ct values were also observed in symptomatic cases vs. controls in 3/7 (43\%) studies, and associated with more severe symptoms in $2 / 2$ studies. Contradictory associations were identified for non-C. difficile bacterial and parasitic pathogens.

Conclusions: In conclusion, some studies reported clinically useful associations between Ct values and patient or healthcare outcomes; additional, well-designed, large-scale trials are warranted based on these findings.

Systematic Review Registration: [PROSPERO], identifier [CRD42020167239].

Keywords: cycle threshold, pathogen load, gastrointestinal pathogens, systematic review, qPCR, clinical outcomes

\section{INTRODUCTION}

Gastrointestinal infections contribute significantly to the burden of illness from infectious diseases worldwide $(1,2)$. Rotavirus is the principal cause of diarrhea mortality, responsible for a high attributable fraction among all age groups (13.9\%) (3). Shigella, the second most common cause of diarrhea mortality, is a key contributor to diarrheal death among children younger than 5 years (14.3\%), mainly in low income countries (3).

Quantitative $(\mathrm{q})$ polymerase chain reaction (PCR) is a robust and increasingly common methodology for rapid syndromic testing due to its sensitivity and specificity for identification of pathogens. In infectious diseases, qPCR cycle threshold (Ct) values represent the number of amplification cycles required for the fluorescent signal to exceed the basal threshold level. $\mathrm{Ct}$ values are inversely related to the number of copies of the target gene in a sample, meaning that lower $\mathrm{Ct}$ values correlate with higher pathogen loads. In infectious diseases, qPCR Ct values have potential utility in providing clinicians with information regarding genomic load that may help guide clinical and infection-control decisions. In addition, Ct values may help to clarify diagnostic uncertainty in cases where there is difficulty interpreting binary results, for example when distinguishing between causative infectious pathogen and asymptomatic carriage/colonization (4-6), particularly as identification of multiple pathogens is common $(7,8)$.

Notably, unprecedented challenges from the COVID-19 pandemic have raised the interest in clinical and diagnostic utility of Ct values $(9,10)$. However, in a recent systematic review of the utility of $\mathrm{Ct}$ values in respiratory infections (parallel to this study), no universal conclusions could be reached [In press: J Antimicrob Chemother 2021]. This systematic review assesses the global medical literature for associations between $\mathrm{Ct}$ values of gastrointestinal pathogens and patient or healthcare outcomes.

\section{METHODS}

This systematic review was undertaken according to the principles outlined in the Cochrane handbook and guidance published by the Center for Reviews and Dissemination. The original protocol was published in the PROSPERO database (CRD42020167239) and included broad search terms unrestricted by pathogen or disease type. This review focuses on gastrointestinal pathogens.

\section{Eligibility Criteria}

Literature searches of MEDLINE, EMBASE, and the Cochrane Library using search tools at ncbi.nlm.nih.gov/pubmed, embase.com and cochranelibrary.com were undertaken to identify studies reporting on the presence or absence of an association between qPCR Ct values and patient or healthcare outcomes (see Supplementary Table 1 for the PubMed search strategy). The search strategy comprised three concepts: (real-time [rt]-PCR OR qPCR) AND Ct values AND pathogen. Randomized-controlled, single-arm, nonrandomized comparative and observational (retrospective or prospective) studies were included. Animal studies, systematic reviews, non-systematic reviews and meta-analyses were excluded; however, additional publications were identified by manual citation searching of appropriate reviews. Searches were limited to English language studies, for reasons of feasibility.

\section{Study Selection and Data Extraction}

Titles and abstracts were screened, based on eligibility criteria, for inclusion by two independent reviewers who then assessed the full texts of relevant studies; a third reviewer resolved conflicts. Key data from all included studies were captured by one reviewer, and subsequently verified by another reviewer. Outcomes were broadly divided into the following categories: mortality, symptomatic vs. asymptomatic, severity of symptoms, duration of symptoms, intensive care unit (ICU) admission, hospitalization and length of stay (LOS).

The quality and risk of bias of each study was assessed using a tool relevant for each study design (Newcastle Ottawa Scale for cross-sectional, cohort, and case-control studies (11). 


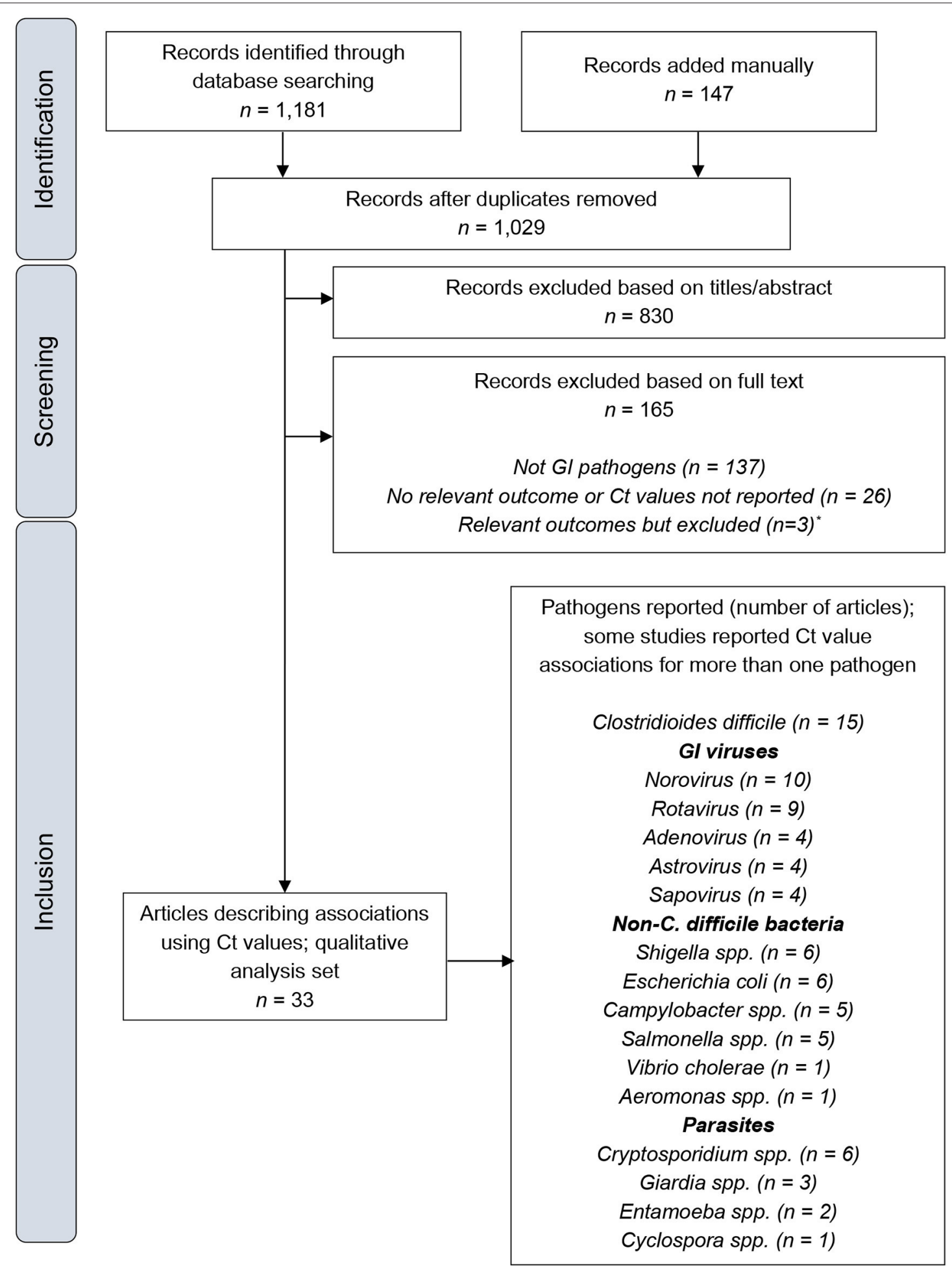

FIGURE 1 | PRISMA flow diagram. Ct, cycle threshold; Gl, gastrointestinal. *Details of these publications are provided in the Supplementary Material. 


\section{RESULTS}

\section{Overview of Studies Included}

Literature searches, conducted January 14-17, 2020, identified 1,029 unique records. Application of distinct screening and restriction parameters specific to gastrointestinal infections identified 33 eligible studies. Most studies reported $\mathrm{Ct}$ value association for more than one pathogen; the most commonly studied pathogens were Clostridioides difficile $(n=15)$, norovirus $(n=10)$, and rotavirus $(n=9)$ (Figure 1). All studies identified gastrointestinal pathogens from stool samples. In studies of $C$. difficile, the majority used genes encoding toxin $\mathrm{A}$ or $\mathrm{B}$ as PCR targets.

The majority of outcomes reported were related to symptoms, including symptom severity, symptomatic vs. asymptomatic and duration of symptoms. Mortality was assessed by three studies. No studies investigated associations between Ct values and hospitalization and/or ICU admission. The majority $(84.8 \%$; $28 / 33$ ) of studies did not report normalized Ct values. Some $(66.7 \% ; 22 / 33)$ studies presented Ct value distributions.

\section{Quality and Bias}

Using Newcastle-Ottawa scales, all cross-sectional studies, cohort studies and case-control studies were classed as being of poor quality (Supplementary Tables 2-4, respectively). This was generally due to a lack of comparability between groups, insufficient or unjustified sample sizes, the use of nonrepresentative samples (often hospitalized patients or agespecific populations) and a lack of detail regarding patient follow-up or non-response; ascertainment of exposure and outcome was usually appropriate. In an assessment of qPCR methodology, 14/29 (48\%) full-length articles were considered to have some or many gaps in the reported methodology (Supplementary Table 5).

\section{Clostridioides difficile}

C. difficile was the most commonly reported gastrointestinal pathogen with respect to articles describing associations between $\mathrm{Ct}$ values and patient or healthcare outcomes (Table 1). Two studies investigated the association of Ct value with mortality, of which one $(N=1,013)$ reported no significant associations (12). The second study, conducted by Davies et al. at four UK hospitals, was the largest $C$. difficile assessment in this systematic review $(N=1,281)$. The authors reported significantly lower median $\mathrm{Ct}$ values for patients who died with $C$. difficile infection compared with those who survived [25.5 $(n=123)$ vs. $27.5(n=762)$, respectively; $p=0.021$ ] (13).

Among 12 articles reporting associations between Ct values and symptoms, eight investigated severity of symptoms. Three studies reported significantly lower median Ct values in patients with severe or complicated disease vs. those with mild/moderate disease: De Francesco et al. $(N=421)$ severe $25.9(n=199)$ vs. mild/moderate $28.1(n=222), p=0.00001$; Jazmati et al. $(N=$ 99) severe 26.5 vs. mild/moderate $31.2, p=0.02$; Kamboj et al. $(N=183)$ severe 24.5, complicated 22.5, and non-severe 28.0, $p=0.005$ (14-16). Jazmati et al. further described lower Ct values as a predictor of severe disease [area under the receiver operating characteristic curve $0.77,95 \%$ confidence interval (CI) $0.62-0.92$; $p=0.013$ ] (14). Reigadas et al. $(n=299)$ showed that Ct value was independently associated with poor outcome $(p<0.001)$ and classified patients into risk categories accordingly; high risk of poor outcome (median $\mathrm{Ct}<23.5$ ); medium risk of poor outcome (median Ct 23.5-27.9); and low risk of poor outcome (median $\mathrm{Ct} \geq 28.0$ ) (17). A further three studies with numbers of PCRpositive patients ranging from 62 to 219 , reported lower $\mathrm{Ct}$ values in patients with poorer outcomes or more severe disease; however, differences did not reach statistical significance (18-20).

Four studies investigated differences in $\mathrm{Ct}$ values in case vs. control subjects. In Crobach et al. $(N=208)$ mean quantification cycle (Cq) values were significantly lower $(p<0.001)$ in symptomatic patients who were toxin A/B-positive by enzyme immunoassay (24.4, 95\% CI 23.5-25.3) than symptomatic patients who were toxin A/B-negative (30.4, 95\% CI 29.5-31.3) and asymptomatic carriers (29.2, 95\% CI 27.3-31.2) (5). Similar observations were reported in pediatric patients by Bub et al. $(N=13$; median Ct 32 in symptomatic cases vs. 36 in controls, no significance reported $)$ and Hecht et al. $(N=193$; median Ct 23.8 in true infections vs. 30.5 in colonized, $p=0.03)(6,21)$. In a study $(n=85)$ by Bruijnesteijn van Coppenraet et al., although no significant difference in Ct values were observed between cases and controls across all subjects, Ct values were significantly lower in cases vs. controls for age group 21-50 years (22).

Two studies $(N \leq 120)$ investigated association of $\mathrm{Ct}$ value with duration of symptoms; no significant associations were reported in either study $(23,24)$. One large study $(N=1,281)$ of diarrheal patients in the UK investigated Ct value and LOS; however, no significant associations were reported, except for patients with PCR-ribotype 027, where LOS was significantly increased in those with low vs. high Ct value (32.5 vs. 28 days; $p=0.018)(13)$.

\section{Gastrointestinal Viruses}

Associations between patient or healthcare outcomes and the $\mathrm{Ct}$ value of gastrointestinal viruses were investigated in 14 studies, with the most commonly studied viruses being norovirus and rotavirus ( $n=10$ and $n=9$, respectively) (Table 2 ). The majority of studies $(n=10)$ investigated the difference in $\mathrm{Ct}$ values (or viral load derived from Ct values) between cases and controls (symptomatic and asymptomatic, or patients with or without diarrhea).

In general, norovirus, particularly norovirus genogroup II (GII), infections were found to have significantly lower median $\mathrm{Ct}$ values in infections vs. controls. Kabue et al. $(N=$ 122) reported that lower median $\mathrm{Ct}$ values were observed in symptomatic pediatric patients compared with asymptomatic pediatric patients infected with norovirus GII $(n=104 ; 27.0$ vs. 34.6; $p=0.0009$ ) (25). Similar outcomes were reported in Kabayiza et al. ( $n=51 ; 25.8$ vs. $29.5 ; p=0.04)$, Phillips et al. $(n=589 ; 34$ vs. $37 ; p<0.0001)$, Saito et al. $(n=$ 467 ; 26.4 vs. $30.1 ; p=0.0001)$, and Dung et al. $(n=138$; $6.85 \log$ copies/ml vs. $5.07 \log$ copies/ml; $p=0.02)(4,26-28)$. 
TABLE 1 | Summary of studies that assessed PCR Ct values for C. difficile infections against patient clinical presentation and outcomes.

Outcome Study $\begin{gathered}\text { Number of Population } \\ \text { PCR+ patients }\end{gathered}$

\begin{tabular}{lll}
\hline Mortality & $\begin{array}{l}\text { Davies et al. Plos One } \\
2018\end{array} \quad 1,281 \quad$ UK, hospital
\end{tabular}

- Lower median Ct values for patients who died $(n=123)$ with infection compared with those who survived ( $n=762$; median $C t 25.5$ and 27.5, respectively; $p=0.021$ )

- Following optimal cut-off determination, low $\mathrm{Ct}$ was defined as $\leq \mathbf{2 5}$ and was significantly associated with mortality $(p=0.032)$

\begin{tabular}{|c|c|c|c|c|}
\hline & Rao et al. CID 2015 & 1,013 & $\begin{array}{l}\text { USA, hospital, } \\
\geq 18 \text { years }\end{array}$ & $\begin{array}{l}\text { - There was no association between Ct values and 30-day mortality [OR } 1(95 \% \mathrm{Cl} \\
0.93-1.08) ; p=0.95]\end{array}$ \\
\hline $\begin{array}{l}\text { Severity of } \\
\text { symptoms }\end{array}$ & Rao et al. CID 2015 & 1,013 & $\begin{array}{l}\text { USA, hospital, } \\
\geq 18 \text { years }\end{array}$ & $\begin{array}{l}\text { - There was no association between Ct values and severe CDI [OR } 1.01(95 \% \mathrm{Cl} \\
0.93-1.09) ; p=0.873]\end{array}$ \\
\hline
\end{tabular}

De Francesco et al.

$421 \quad$ Italy, hospital and Anaerobe 2019
Origüen et al. JCM 2019

(1)

Kamboj et al. J Infect 2018

183

Reigadas et al. J Antimicrob Chemother 2016
Derivation cohort: Spain, hospital, 129 Validation $\geq 17$ years cohort: 170
Spain, hospital, $\geq 18$ years
- Ct values <25 were significantly associated with severe disease vs. $\mathrm{mild} / \mathrm{moderate}$ disease [97 (55\%) vs. $79(44 \%) ; p=0.0075$ ]

- Ct values > 25 were significantly associated with $\mathrm{mild} / \mathrm{moderate}$ disease vs. severe disease [143 $(58.3 \%)$ vs. $102(41.6 \%) ; p=0.004]$

- The median Ct values of tcdB PCR in patients with mild/moderate disease were significantly higher (28.1; IQR 7.7) than in patients with severe disease (25.9; IQR 5.9) $(p=0.00001)$

- A Ct value $\leq \mathbf{2 6}$ was significantly associated with patients with a severe disease

- The mean PCR Ct was lower for patients with a poor outcome (24.9 \pm 4.24 vs. $26.05 \pm$ $4.47 ; p=0.07$ ) ("Poor outcome" was defined as the occurrence of a severe or severecomplicated first CDI episode and/or all-cause death within the first 8 weeks after the end of treatment)

- The optimal cut-off Ct value was established as 27.55 , yielding a sensitivity of $78.6 \%$ (95\% Cl 67.1-87.5), a specificity of 35.7\% (95\% Cl 28.2-43.7), a PPV of 35.3\% (95\% Cl 31.5-39.2), and an NPV of 78.9\% (95\% Cl 69.5-85.9)

- Severe and complicated infections were associated with lower Ct values than non-severe infections [median Ct values for non-severe Ct $=28.0(n=168)$, severe $\mathrm{Ct}=24.5(n=11)$, and complicated $\mathrm{Ct}=22.5(n=4) ; p=0.005]$

Derivation cohort

- Ct value was independently associated with poor outcome by multivariate analysis [OR $0.701(95 \% \mathrm{Cl} 0.604-0.813) ; p<0.001$ ]

- Patients were classified into risk categories; high risk of poor-outcome (Ct <23.5 cycles); medium risk of poor-outcome (Ct 23.5-27.9 cycles); and low risk of poor-outcome (Ct $\geq 28.0$ cycles). The sensitivity of the rule was $46.5 \%$ (95\% Cl 32.5-61.1) and specificity was 98.8\% (95\% Cl 93.7-99.8), the PPV was 95.2\% (95\% Cl 86.1-100) and NPV was $78.7 \%$ (95\% Cl 70.9-86.4); the diagnostic accuracy was 81.4\% (95\% Cl 74.7-88.1)

- Patients with poor-outcome CDI episodes had lower median Ct values than those without poor-outcome CDI episodes (24.8 vs. 28.9; $p<0.001$ )

Validation cohort

- Median Ct value was lower for episodes with poor outcome than favorable (21.9 vs. 27.0; $p<0.001$ )

- Independent association between $\mathrm{Ct}$ value and poor outcome $(p<0.001)$ and the high-risk category $(\mathrm{Ct}<23.5)$ and poor outcome $(p<0.001)$

Anikst et al. Diag

Microbiol Infect Dis

$118 \quad$ USA $^{*}$, adults

- No difference in organism burden between groups with $(n=59)$ and without $(n=59)$ clinically significant diarrhea [median Ct, 26.9; (IQR 23.9-32.2) vs. 27.1 (IQR 23.4-30.7); $p=0.25$; mean Ct 27.9 vs. 27.4]

Jazmati et al. Clin

Microbiol Infect 2016

Germany, hospital - Patients with severe disease had significantly lower Ct values compared with non-severe infections [26.5 $\pm 4.8,(n=9)$ vs. $31.2 \pm 4.8(n=45) ; p=0.02]$, describing lower $\mathrm{Ct}$ values as a predictor of severe disease (area under the receiver operating characteristic curve $0.77,95 \% \mathrm{Cl} 0.62-0.92 ; p=0.013$ )

Sante et al. Enferm Infecc Microbiol Clin 2018

$62 \quad$ Spain, hospital

- The was no significant difference between Ct values of patients with and those without serious disease [27 \pm 4 ( $n=42)$ vs. $29 \pm 14(n=20)$, respectively; $p=0.23$ ]

\begin{tabular}{|c|c|c|c|}
\hline $\begin{array}{l}\text { Case vs. } \\
\text { control }\end{array}$ & $\begin{array}{l}\text { Crobach et al. J Clin } \\
\text { Microbiol } 2018\end{array}$ & 208 & $\begin{array}{l}\text { Netherlands, } \\
\text { hospital }\end{array}$ \\
\hline
\end{tabular}

- Comparable mean Ct values were observed for symptomatic patients with subsequent negative toxin A/B immunoassay results [30.4 (95\% Cl 29.5-31.3)] and asymptomatic carriers [29.2 (95\% Cl 27.3-31.2)], while symptomatic patients with positive toxin A/B results had significantly lower mean Ct values, according to ANOVA [24.4 (95\% Cl 23.5-25.3); $p<0.001]$ 
TABLE 1 | Continued

\begin{tabular}{|c|c|c|c|c|}
\hline Outcome & Study & $\begin{array}{l}\text { Number of } \\
\text { PCR+ patients }\end{array}$ & Population & Outcome measure (significant associations bolded) \\
\hline & $\begin{array}{l}\text { Hecht et al. Open } \\
\text { Forum Infect Dis } 2019\end{array}$ & 193 & USA $^{\star},<3$ years & $\begin{array}{l}\text { - Among six (4\%) patients who met strict criteria for true infection (including } \\
\text { consistent clinical syndrome with no alternative etiology of diarrhea), median } \\
\text { Ct value (IQR) was significantly lower than those who did not meet the criteria } \\
\text { (classed as colonized) } 23.8(22.0-29.5) \text { vs. } 30.5(26.3-35.8), p=0.03\end{array}$ \\
\hline & $\begin{array}{l}\text { Bruijnesteijn van } \\
\text { Coppenraet et al. Clin } \\
\text { Microbiol Infect } 2015\end{array}$ & 85 & $\begin{array}{l}\text { Netherlands, } \\
\text { primary Care }\end{array}$ & $\begin{array}{l}\text { - No significant difference in Ct values across all case subjects and controls; however, Ct } \\
\text { values were significantly lower }(p<0.05) \text { in cases vs. controls for patients aged } 21-50 \\
(n=19)\end{array}$ \\
\hline & $\begin{array}{l}\text { Bub et al. Tropical } \\
\text { Medicine and } \\
\text { International Health } \\
2017\end{array}$ & 13 & Ivory Coast & $\begin{array}{l}\text { - There was a trend toward lower median Ct values in symptomatic patients vs. controls } \\
\text { (32 vs. 36, respectively) }\end{array}$ \\
\hline \multirow[t]{2}{*}{$\begin{array}{l}\text { Duration of } \\
\text { symptoms }\end{array}$} & $\begin{array}{l}\text { Feghaly et al. CID } \\
2013 a\end{array}$ & 120 & $\begin{array}{l}\text { USA, hospital, } \\
\text { adult }\end{array}$ & $\begin{array}{l}\text { - When patients were segregated into quartiles based on their initial Ct values, there was } \\
\text { no difference in the time to diarrhea resolution among patients }\end{array}$ \\
\hline & $\begin{array}{l}\text { Feghaly et al. J Ped } \\
2013 b\end{array}$ & 74 & $\begin{array}{l}\text { USA, hospital, } \\
\text { pediatric }\end{array}$ & $\begin{array}{l}\text { - When patients were segregated into quartiles based on their initial Ct values, there was } \\
\text { a paradoxical trend toward a longer interval to diarrhea resolution in children with a lower } \\
\text { bacterial burden at diagnosis }(p=0.06) \\
\text { - Lower fecal bacterial burden at diagnosis (as calculated by Ct value) was associated } \\
\text { with longer times to diarrhea resolution (HR 0.93; } 95 \% \mathrm{Cl} 0.86-1 ; p=0.058 \text { ) }\end{array}$ \\
\hline Recurrence & $\begin{array}{l}\text { Origüen et al. JCM } \\
2019\end{array}$ & 219 & $\begin{array}{l}\text { Spain, hospital, } \\
\geq 18 \text { years }\end{array}$ & $\begin{array}{l}\text { - The mean } \mathrm{Ct} \text { value was lower in patients with recurrence compared with those } \\
\text { without }(24.00 \pm 3.28 \text { vs. } 26.02 \pm 4.54 ; p=0.002)\end{array}$ \\
\hline Median LOS & $\begin{array}{l}\text { Davies et al. Plos One } \\
2018\end{array}$ & 1,281 & UK, hospital & $\begin{array}{l}\text { - Patients with low Ct values }(\leq 25) \text { had a numerically greater LOS compared with those } \\
\text { with high Ct values ( }>25) \text {; however this difference was not significant (Ct } \leq 25: 28 \text { days } \\
\text { vs. Ct }>25: 23 \text { days; } p=0.77) \\
\text { - In patients with presence of PCR-ribotype } \mathbf{0 2 7} \text {, LOS was significantly increased } \\
\text { in those with low vs. high Ct ( } \mathbf{3 2 . 5} \text { days vs. } \mathbf{2 8} \text { days; } \boldsymbol{p}=\mathbf{0 . 0 1 8 )}\end{array}$ \\
\hline \multirow[t]{2}{*}{$\begin{array}{l}\text { Other } \\
\text { biomarker }\end{array}$} & $\begin{array}{l}\text { Davies et al. Plos One } \\
2018\end{array}$ & 1,281 & UK, hospital & 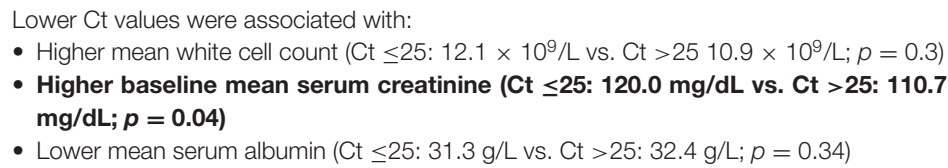 \\
\hline & $\begin{array}{l}\text { De Francesco et al. } \\
\text { Anaerobe } 2019\end{array}$ & 421 & $\begin{array}{l}\text { Italy, hospital and } \\
\text { community }\end{array}$ & $\begin{array}{l}\text { - Statistically significant correlation between low } \text { Ct values and leucocytosis } \\
(p<0.001) \text { but not with the alteration in baseline creatinine or serum albumin level }\end{array}$ \\
\hline
\end{tabular}

Text in bold indicates a statistically significant association.

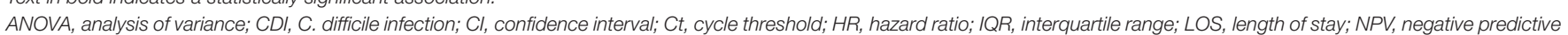
value; OR, odds ratio; PCR, polymerase chain reaction; PPV, positive predictive value; UK, United Kingdom; USA, United States of America.

* Likely setting although not confirmed in source material.

Additionally, Liu et al. reported a pathogen quantity-dependent association with diarrhea in children $<5$ years old (29). Elfving et al. also reported lower median $\mathrm{Ct}$ values in patients vs. controls, but the difference was not significant (25.1 vs. 26.9; $p=0.28)(30)$.

One study investigated Ct values of norovirus GII and fatal outcomes $(n=534)$ and found no association (31). One other study reported no significant associations between $\mathrm{Ct}$ values and symptom duration $(n=623)$ or infectiousness $(n=110)$ in patients with infections caused by norovirus (32).

Similar to norovirus, multiple studies showed significantly lower $\mathrm{Ct}$ values (or $\mathrm{Cq}$ ) in cases of symptomatic rotavirus infection vs. controls. Phillips et al. $(N=153)$ reported lower median $\mathrm{Ct}$ values in rotavirus intestinal infections vs. controls (18 vs. 37; $p<0.0001)$ (33). Dung et al. $(n=113)$ reported significantly higher median viral loads in children with diarrhea compared with those without (10.6 log copies/ml vs. $8.33 \log$ copies/ml; $p<0.001$ ) (26), and one study in children $<5$ years by Liu et al. reported strong pathogen quantity-dependent associations with diarrhea (29). Supporting these observations, Kabayiza et al. $(n=325)$ reported that lower median $\mathrm{Ct}$ values were significantly associated with more severe symptoms, including vomiting, severe dehydration and intravenous fluid therapy, in patients with infections caused by rotavirus (27). Kang et al. $(N=91)$ also reported significant associations between severe diarrhea and low Ct values (reported as "crossing points") (34). Four further studies also reported lower median $\mathrm{Ct}$ values in patients vs. controls/asymptomatic patients, but differences did not reach statistical significance: Elfving et al. ( $n=19 ; 24.4$ vs. $26.0 ; p=0.50)$; Kabayiza et al. $(n=238 ; 21.16$ vs. $23.29 ; p=0.24)$; Ramani et al. $(n=103 ; 26.26$ vs. $27.34 ; p=0.087)$ and Mukhopadhya et al. $(n=15 ; 17.21$ vs. $30.98 ; p=0.086)(27,30,35,36)$. Notably, adjustment of an outlier in the study by Mukhopadhya et al. resulted in the difference reaching statistical significance $(p=0.007)$ (36). 
TABLE 2 | Summary of studies that assessed PCR Ct values for gastrointestinal viruses against patient clinical presentation and outcomes.

\begin{tabular}{|c|c|c|c|c|c|c|c|c|}
\hline Outcome & Study & Pathogen(s) & $\begin{array}{l}\text { Number of } \\
\text { PCR+ patients }\end{array}$ & Population & \multicolumn{4}{|c|}{ Outcome measure } \\
\hline \multicolumn{9}{|c|}{ NOROVIRUS } \\
\hline Mortality & $\begin{array}{l}\text { Gustavsson et al. } \\
\text { J Clin Virol } 2015\end{array}$ & Norovirus & $534^{*}$ & $\begin{array}{l}\text { Sweden, hospital, } \\
>60 \text { years }\end{array}$ & \multicolumn{4}{|c|}{$\begin{array}{l}\text { - Ct values were not associated with fatal outcomes [46 patients } \\
\text { deceased; HR } 0.97 \text { (95\% Cl 0.92-1.02) per Ct unit decrease; } p=0.17 \text { ] }\end{array}$} \\
\hline \multirow[t]{6}{*}{$\begin{array}{l}\text { Severity of } \\
\text { symptoms }\end{array}$} & \multirow{2}{*}{\multicolumn{2}{|c|}{$\begin{array}{l}\text { Kabayiza et al. Clin Norovirus } \\
\text { Microbiol and Infec } \\
2014 b\end{array}$}} & \multirow[t]{2}{*}{98} & $\begin{array}{l}\text { Rwanda, } \\
\text { community and } \\
\text { hospital, } \leq 5 \text { years }\end{array}$ & \multicolumn{4}{|c|}{$\begin{array}{l}\text { - No significant difference in Ct values was observed for norovirus Gl or } \\
\text { Gll for clinical markers including vomiting, dehydration or intravenous } \\
\text { fluid use }\end{array}$} \\
\hline & & & & & & Vomiting: Yes/No & $\begin{array}{l}\text { Dehydration: } \\
\text { Severe/moderate/ } \\
\text { mild }\end{array}$ & $\begin{array}{l}\text { IV Fluids: } \\
\text { Yes/No }\end{array}$ \\
\hline & & \multirow[t]{2}{*}{ Norovirus Gl } & \multirow[t]{2}{*}{22} & & OR & $\begin{array}{c}1.80 \\
p=0.35\end{array}$ & $\begin{array}{c}0.33 \\
p=0.77\end{array}$ & $\begin{aligned} 2.08 \\
p=0.13\end{aligned}$ \\
\hline & & & & & Ct & $\begin{array}{l}30.2 / 31.7 \\
p=0.91\end{array}$ & $\begin{array}{l}34.0 / 29.4 / 31 / 0 \\
\quad p=0.69\end{array}$ & $\begin{array}{l}30.2 / 28.3 \\
p=0.37\end{array}$ \\
\hline & & \multirow[t]{2}{*}{ Norovirus Gll } & \multirow[t]{2}{*}{76} & & OR & $\begin{array}{c}0.84 \\
p=0.51\end{array}$ & $\begin{array}{c}0.69 \\
p=0.16\end{array}$ & $\begin{array}{c}0.66 \\
p=0.09\end{array}$ \\
\hline & & & & & $\mathrm{Ct}$ & $\begin{array}{l}27.7 / 28.6 \\
p=0.83\end{array}$ & $\begin{array}{l}30.4 / 27.2 / 28.4 \\
p=0.54\end{array}$ & $\begin{array}{l}27.0 / 28.5 \\
p=0.54\end{array}$ \\
\hline \multirow[t]{11}{*}{$\begin{array}{l}\text { Case versus } \\
\text { control }\end{array}$} & $\begin{array}{l}\text { Liu et al. Lancet } \\
2016\end{array}$ & Norovirus GII & $5,304^{\dagger}$ & $\begin{array}{l}\text { Bangladesh, India, } \\
\text { Pakistan, The } \\
\text { Gambia, Kenya, } \\
\text { Mali and } \\
\text { Mozambique, } \\
\text { community, <5 } \\
\text { years }\end{array}$ & \multicolumn{4}{|c|}{$\begin{array}{l}\text { - Norovirus Gll showed associations with diarrhea } \\
\text { - Cq values }<27.6 \text { were defined as "diarrhea-associated" as the } 95 \% \mathrm{Cl} \\
\text { or the OR was }>1 \text {. Ct values }<23.4 \text { were defined as "highly } \\
\text { diarrhea-associated" as the } 95 \% \mathrm{Cl} \text { or the OR was }>2 \text {. The ROC } \\
\text { cut-off maximally discriminating case-control status was a Ct value of } \\
28.8 \text { (Youden Index 0.15) }\end{array}$} \\
\hline & $\begin{array}{l}\text { Saito et al. CID } \\
2014\end{array}$ & Norovirus & $\begin{array}{c}607 \text { (140 Gl, } 460 \\
\text { Gll, } 7 \text { Gl/II) } \\
\text { infections from } \\
409 \text { patients }\end{array}$ & $\begin{array}{l}\text { Peru, community, } \\
\text { infants }\end{array}$ & \multicolumn{4}{|c|}{$\begin{array}{l}\text { Median Ct values were lower in diarrheal compared with } \\
\text { non-diarrheal samples [orovirus Gl: } 28.2 \text { vs. } 31.0(p<0.066) \text {; } \\
\text { norovirus Gll: } 26.4 \text { vs. } 30.1(p=0.0001) \text {, respectively] }\end{array}$} \\
\hline & $\begin{array}{l}\text { Phillips et al. BMC } \\
\text { Infect Dis 2009a }\end{array}$ & Norovirus Gll & 589 & $\begin{array}{l}\text { England, } \\
\text { community/ } \\
\text { primary care }\end{array}$ & \multicolumn{4}{|c|}{$\begin{array}{l}\text { - The median rt-PCR Ct value was significantly lower in infectious } \\
\text { intestinal disease cases vs. control }\end{array}$} \\
\hline & & & & & & $\begin{array}{l}\text { Median }[\mathrm{IQR}] \mathrm{Ct} \\
\text { value: cases }\end{array}$ & $\begin{array}{l}\text { Median }[\mathrm{IQR}] \mathrm{Ct} \\
\text { value: controls }\end{array}$ & $p$-value \\
\hline & & & & & All ages & $34(25-37)$ & $38(35-39)$ & $<0.0001$ \\
\hline & & & & & $<5$ years & 34 (26-37) & 37 (34-48) & $<0.0001$ \\
\hline & & & & & $\geq 5$ years & 34 (25-38) & 38 (36-39) & $<0.0001$ \\
\hline & $\begin{array}{l}\text { Dung et al. J Virol } \\
\text { Methods } 2012\end{array}$ & Norovirus Gll & 138 & $\begin{array}{l}\text { Vietnam, hospital, } \\
\leq 60 \text { months }\end{array}$ & \multicolumn{4}{|c|}{$\begin{array}{l}\text { - Results are presented in log of target RNA copy number per mL; } \\
\text { however, it is noted that these were converted from Cp values using a } \\
\text { standard curve } \\
\text { - The viral load of norovirus Gll was significantly higher in } \\
\text { samples from children with diarrhea (6.85 log/RNA copies/ml; } \\
\text { range: } 2.89-9.71) \text { than those without (5.07 log/RNA copies/ml; } \\
\text { range: } 3.63-9.16) p=0.02\end{array}$} \\
\hline & $\begin{array}{l}\text { Kabue et al. J Clin } \\
\text { Virol } 2016\end{array}$ & $\begin{array}{l}\text { Norovirus } \\
\text { Norovirus Gl } \\
\text { Norovirus Gll }\end{array}$ & $\begin{array}{l}122 \text { (71 Gll only, } \\
18 \text { Gl only, } 33 \\
\text { Gl/Gll mixed) }\end{array}$ & $\begin{array}{l}\text { South Africa, } \\
\text { clinics } \\
\text { (community), <5 } \\
\text { years }\end{array}$ & \multicolumn{4}{|c|}{$\begin{array}{l}\text { - There was no difference in median Ct value between symptomatic and } \\
\text { asymptomatic patients ( } 28.06 \text { vs. } 27.58 \text {, respectively; } p=0.32) \\
\text { - Significantly lower median Ct values were observed in } \\
\text { symptomatic patients compared with asymptomatic patients } \\
\text { (27.02 vs. } \mathbf{3 4 . 5 9} \text {, respectively; } \boldsymbol{p}=\mathbf{0 . 0 0 0 9 )}\end{array}$} \\
\hline & $\begin{array}{l}\text { Kabayiza et al. } \\
\text { Pediatr Infect Dis J } \\
2014 \text { a }\end{array}$ & $\begin{array}{l}\text { Norovirus Gl and } \\
\text { Gll }\end{array}$ & $\begin{array}{l}\text { Gl, } n=27 \\
\text { Gll, } n=51\end{array}$ & $\begin{array}{l}\text { Rwanda, } \\
\text { community and } \\
\text { hospital, } \leq 5 \text { years }\end{array}$ & \multicolumn{4}{|c|}{$\begin{array}{l}\text { - Significantly lower } \text { Ct values in patients vs. controls for } \\
\text { norovirus Gll (25.79 vs. } 29.54 ; \boldsymbol{p}=\mathbf{0 . 0 4}) \text {, but not for norovirus GI } \\
(29.25 \text { vs. } 28.94 ; p=0.08)\end{array}$} \\
\hline & $\begin{array}{l}\text { Elfving et al. JCM } \\
2014\end{array}$ & Norovirus Gll & 37 & $\begin{array}{l}\text { Zanzibar, } \\
\text { community, } 2 \\
\text { months-5 years }\end{array}$ & \multicolumn{4}{|c|}{$\begin{array}{l}\text { - There was no significant difference in median Ct values between } \\
\text { patients and controls for infections caused by norovirus Gll (25.1 vs. } \\
\text { 26.9, respectively; } p=0.28 \text { ) }\end{array}$} \\
\hline
\end{tabular}


TABLE 2 | Continued

Outcome Study Pathogen(s) $\begin{gathered}\text { Number of } \\ \text { PCR }+ \text { patients }\end{gathered}$ Population

- By multivariate logistic regression analysis (accounting for age and gender), a cut-off Ct value of 45 was associated with disease (OR 10.1; CI 3.5-29.1; $p<0.0001$ )

\begin{tabular}{lllll}
\hline $\begin{array}{l}\text { Duration of } \\
\text { symptoms }\end{array}$ & $\begin{array}{l}\text { Partridge et al. J } \\
\text { Hosp Infect 2012 }\end{array}$ & Norovirus & $623^{\ddagger}$ & UK, hospital \\
\hline $\begin{array}{l}\text { Infectiousness Partridge et al. J } \\
\text { Hosp Infect 2012 }\end{array}$ & Norovirus & 110 & UK, hospital \\
$\begin{array}{l}\text { ROTAVIRUS } \\
\begin{array}{l}\text { Severity of } \\
\text { symptoms }\end{array}\end{array} \begin{array}{l}\text { Kabayiza et al. } \\
\begin{array}{l}\text { Clinc Microbiol } \\
\text { and Infec 2014b }\end{array}\end{array}$ & Rotavirus & 325 & $\begin{array}{l}\text { Rwanda, } \\
\text { community and } \\
\text { hospital, } \leq 5 \text { years }\end{array}$
\end{tabular}

- No significant correlation was identified between duration of symptoms from time of sampling and Ct value of the sample (Spearman rank correlation coefficient: $-0.077 ; p>0.2)$

- There was no significant difference in initial Ct value between onward transmitters and non-transmitters (24.98 vs. 26.56; $p=0.19$ )

- Lower Ct values for rotavirus were significantly associated with multiple clinical markers (vomiting, more severe dehydration and intravenous fluid therapy) in univariate and multivariate analyses Vomiting: Yes/No Dehydration: IV Fluids:
Severe/moderate/mild Yes/No

Univariate analysis

$\begin{array}{cccc}\text { OR } & \mathbf{2 . 8 0} & \mathbf{2 . 4 9} & \mathbf{3 . 7 8} \\ & \boldsymbol{p}<\mathbf{0 . 0 0 0 1} & \boldsymbol{p}<\mathbf{0 . 0 0 0 1} & \boldsymbol{p}<\mathbf{0 . 0 0 0 1} \\ \mathrm{Ct} & \mathbf{2 1 . 2 / 2 2 . 3} & \mathbf{2 0 . 5 / 2 1 . 5 / 2 2 . 8} & \mathbf{2 0 . 8 / 2 3 . 0} \\ & \boldsymbol{p}=\mathbf{0 . 0 3 5} & \boldsymbol{p}=\mathbf{0 . 0 0 8 5} & \boldsymbol{p}=\mathbf{0 . 0 0 0 5} \\ & \text { Vomiting: Yes/No } & \text { Severe } & \text { IV Fluids: } \\ & & \text { dehydration: } & \text { Yes/No } \\ & & \text { Yes/No } & \end{array}$

Age-adjusted multivariate analysis

$$
\begin{array}{cccc}
\mathrm{OR}(\mathrm{Cl}) & 1.57(1.04-2.33) & 1.47(0.94-2.44) & 2.18 \\
& p=0.032 & p=0.09 & (1.54-3.11) \\
& & & p<0.0001
\end{array}
$$

Kang et al. J Med Rotavirus A $\quad 91 \quad$ India, hospital and $\bullet$ There was a significant negative correlation $(\boldsymbol{r}=-\mathbf{0 . 8 0}$; Virol $2004 \quad$ community, $p<0.001$ ) between symptom severity and the crossing point (Ct pediatric value) on the assay, indicating that children with more severe diarrhea have higher viral loads than children with less severe disease

- Mean crossing points (Ct values) in children with high Vesikari severity scores (10-15) were 11.7 (95\% Cl 10.5-12.9), low severity scores (3-9) were 26.8 (95\% Cl 24.6-29.0), and asymptomatic children were 35.7 (95\% Cl 32.5-39.0)

- Significant associations were observed between crossing points and the maximum number of stools in $24 \mathrm{~h}(p<0.001)$, the maximum number of times vomited in $24 \mathrm{~h}(p=0.001)$, and dehydration $(p=0.02)$

\begin{tabular}{|c|c|c|c|c|c|c|c|c|}
\hline \multirow[t]{6}{*}{$\begin{array}{l}\text { Case vs. } \\
\text { control }\end{array}$} & $\begin{array}{l}\text { Liu et al. Lancet } \\
2016\end{array}$ & Rotavirus & $5,304^{\dagger}$ & $\begin{array}{l}\text { Bangladesh, India, } \\
\text { Pakistan, The } \\
\text { Gambia, Kenya, } \\
\text { Mali and } \\
\text { Mozambique, } \\
\text { community, <5 } \\
\text { years }\end{array}$ & \multicolumn{4}{|c|}{$\begin{array}{l}\text { - Rotavirus had strong Ct-dependent associations with diarrh } \\
\text { - Cq values }<35.0 \text { were defined as "diarrhea-associated" as the } 95 \% \\
\text { or the OR was }>1 \text {. Cq values }<32.6 \text { were defined as "highly } \\
\text { diarrhea-associated" as the } 95 \% \mathrm{Cl} \text { or the OR was }>2 \text {. The ROC } \\
\text { cut-off maximally discriminating case-control status was a Cq value } \\
26.9 \text { (Youden Index 0.48) }\end{array}$} \\
\hline & \multirow[t]{4}{*}{$\begin{array}{l}\text { Phillips et al. J Clin } \\
\text { Virol } 2009 \mathrm{~b}\end{array}$} & \multirow[t]{4}{*}{ Rotavirus A } & \multirow[t]{4}{*}{153 cases } & \multirow[t]{4}{*}{$\begin{array}{l}\text { England, } \\
\text { community/ } \\
\text { primary care }\end{array}$} & \multicolumn{4}{|c|}{$\begin{array}{l}\text { The median rt-PCR Ct value was significantly lower in infect } \\
\text { intestinal disease cases vs. control, both in all ages and whe } \\
\text { the analysis was restricted to children aged }<5 \text { years }\end{array}$} \\
\hline & & & & & & $\begin{array}{l}\text { Median }[\mathrm{IQR}] \mathrm{Ct} \\
\text { value: cases }\end{array}$ & $\begin{array}{l}\text { Median }[\mathrm{IQR}] \mathrm{Ct} \\
\text { value: controls }\end{array}$ & $p$-value \\
\hline & & & & & All age groups & $18(15-30)$ & 37 (33-39) & $<0.0001$ \\
\hline & & & & & $<5$ years & 17 (15-22) & $37(33-40)$ & $<0.0001$ \\
\hline & $\begin{array}{l}\text { Kabayiza et al. } \\
\text { Pediatr Infect Dis J } \\
2014 \mathrm{a}\end{array}$ & Rotavirus & 238 & $\begin{array}{l}\text { Rwanda, } \\
\text { community and } \\
\text { hospital, } \leq 5 \text { years }\end{array}$ & \multicolumn{4}{|c|}{$\begin{array}{l}\text { - No significant difference in median Ct values between patients vs. } \\
\text { controls (21.16 vs. } 23.29 ; p=0.24)\end{array}$} \\
\hline
\end{tabular}

- No significant associations were found between crossing points and duration of diarrhea, vomiting, or fever 
TABLE 2 | Continued

\begin{tabular}{|c|c|c|c|c|c|}
\hline Outcome & Study & Pathogen(s) & $\begin{array}{c}\text { Number of } \\
\text { PCR+ patients }\end{array}$ & Population & Outcome measure \\
\hline & $\begin{array}{l}\text { Dung et al. J Virol } \\
\text { Methods } 2012\end{array}$ & Rotavirus A & 113 & $\begin{array}{l}\text { Vietnam, hospital, } \\
\leq 60 \text { months }\end{array}$ & $\begin{array}{l}\text { - Results are presented in log of target RNA copy number per mL; } \\
\text { however, it is noted that these were converted from Cp values using a } \\
\text { standard curve } \\
\text { - The viral load of rotavirus A was significantly higher in samples } \\
\text { from children with diarrhea (10.6 log/RNA copies/ml; 5.56-12.49) } \\
\text { than from those without (8.33 log/RNA copies/ml; 5.43-10.52) } \\
(\boldsymbol{p}<0.001)\end{array}$ \\
\hline & $\begin{array}{l}\text { Ramani et al. J } \\
\text { Med Virol }\end{array}$ & Rotavirus & 103 & $\begin{array}{l}\text { India, hospital, } \\
\text { neonatal }\end{array}$ & $\begin{array}{l}\text { - The mean Ct value was } 26.26 \text { (SD 3.06) for symptomatic neonates and } \\
27.34 \text { (SD 2.73) for asymptomatic neonates } \\
\text { - There was no significant difference in viral load between symptomatic } \\
\text { and asymptomatic neonates ( } p=0.087 \text { ) } \\
\text { - Neonates with feed intolerance and abdominal distension had } \\
\text { significantly lower Ct values than those with other gastrointestinal } \\
\text { symptoms }(p=0.02)\end{array}$ \\
\hline & $\begin{array}{l}\text { Elfving et al. JCM } \\
2014\end{array}$ & Rotavirus & 19 & $\begin{array}{l}\text { Zanzibar, } \\
\text { community, } 2 \\
\text { months-5 years }\end{array}$ & $\begin{array}{l}\text { - There was no significant difference in median Ct values between } \\
\text { patients and controls for infections caused by rotavirus ( } 24.4 \text { vs. 26.0, } \\
\text { respectively; } p=0.5 \text { ) } \\
\text { - By multivariate logistic regression analysis (accounting for age } \\
\text { and gender), a cut-off Ct value of } \mathbf{4 5} \text { was associated with } \\
\text { disease (OR } \mathbf{5 . 8} \text {; } \mathbf{C l} \mathbf{1 . 7 - 2 0 . 3 ;} \boldsymbol{p}<\mathbf{0 . 0 0 3} \text { ) }\end{array}$ \\
\hline
\end{tabular}

Mukhopadhya Rotavirus 15:10 India, hospital and • The median Cq at presentation in symptomatic children was 17.21 (IQR

et al. J Med Virol 2013 symptomatic and community, $<5$

5 asymptomatic years
14.36-23.96) compared with 30.98 (IQR 29.38-31.50) in asymptomatic children $(p=0.086)$

- Once removing an outlier in the asymptomatic group, the difference between the initial shedding between symptomatic and asymptomatic samples was found to be statistically significant $(p=0.007)$

\section{ALL OTHER GASTROINTESTINAL VIRUSES}

\section{Severity of Kabayiza et al. Clin}

symptoms Microbiol and Infec

2014b
Rwanda,

community and

hospital, $\leq 5$ years

- No significant associations between Ct values and clinical markers were observed for adenovirus, astrovirus or sapovirus

(1)

Adenovirus

Astrovirus

36

Sapovirus

33

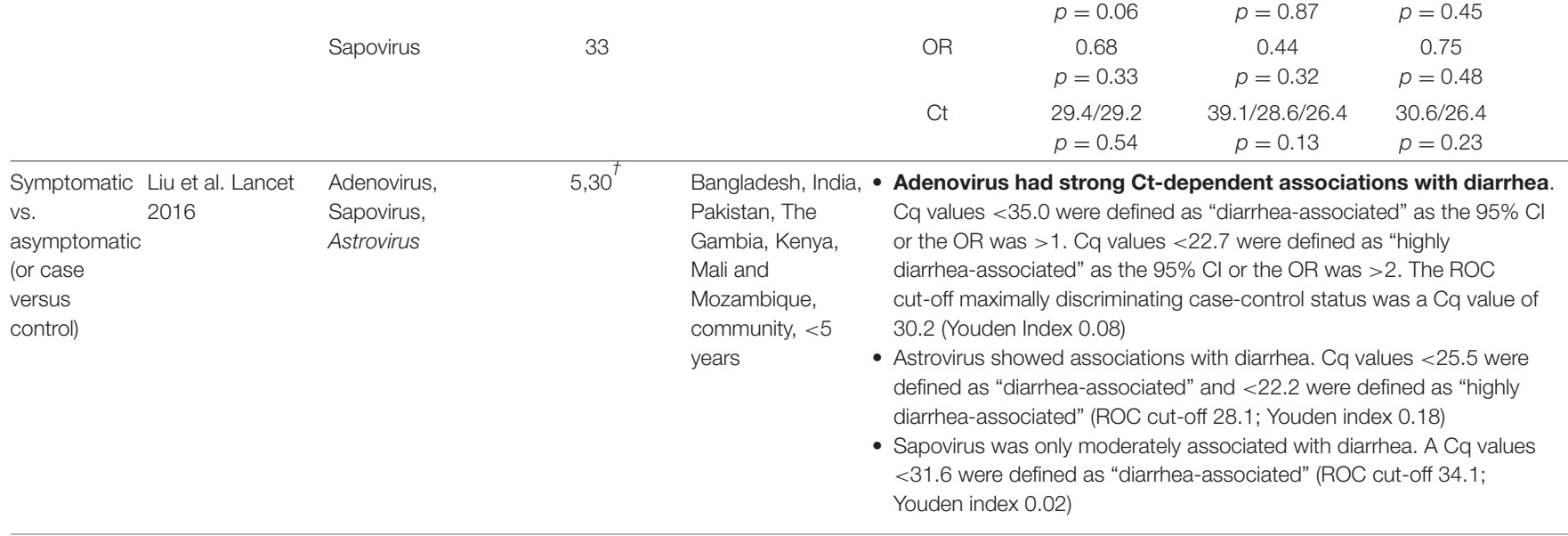


TABLE 2 | Continued

\begin{tabular}{|c|c|c|c|c|c|c|c|}
\hline \multirow[t]{2}{*}{ Outcome } & \multirow{2}{*}{$\begin{array}{l}\text { Study } \\
\text { Kabayiza et al. } \\
\text { Pediatr Infect Dis J } \\
2014 a\end{array}$} & \multirow{2}{*}{ Pathogen(s) } & \multirow{2}{*}{$\begin{array}{c}\text { Number of } \\
\text { PCR+ patients }\end{array}$} & \multirow{2}{*}{$\begin{array}{l}\text { Population } \\
\text { Rwanda, } \\
\text { community and } \\
\text { hospital, } \leq 5 \text { years }\end{array}$} & \multicolumn{3}{|c|}{ Outcome measure } \\
\hline & & & & & \multicolumn{3}{|c|}{$\begin{array}{l}\text { - No significantly lower Ct values in patients vs. controls for other } \\
\text { gastrointestinal viruses }\end{array}$} \\
\hline & & & & & $\begin{array}{l}\text { Median Ct for } \\
\text { patients }\end{array}$ & $\begin{array}{l}\text { Median Ct for } \\
\text { controls }\end{array}$ & $p$-value $(\mathrm{PCR}+)$ \\
\hline & & Adenovirus & 284 & & 36.27 & 35.89 & 0.57 \\
\hline & & Astrovirus & 31 & & 25.79 & 24.15 & 0.31 \\
\hline & & Sapovirus & 38 & & 25.59 & 26.42 & 0.94 \\
\hline & \multirow{2}{*}{\multicolumn{2}{|c|}{$\begin{array}{l}\text { Elfving et al. JCM } \\
2014\end{array}$}} & & \multirow[t]{2}{*}{$\begin{array}{l}\text { Zanzibar, } \\
\text { community, } 2 \\
\text { months-5 years }\end{array}$} & \multicolumn{3}{|c|}{$\begin{array}{l}\text { - There was no significant difference in median Ct values between } \\
\text { patients and controls for infections caused by gastrointestinal viruses }\end{array}$} \\
\hline & & & & & $\begin{array}{l}\text { Median Ct for } \\
\text { patients }\end{array}$ & $\begin{array}{l}\text { Median Ct for } \\
\text { controls }\end{array}$ & $p$-value \\
\hline & & Adenovirus (any) & 98 & & 38.2 & 39.3 & 0.05 \\
\hline & & Adenovirus 40/41 & 16 & & 36.6 & 35.0 & 0.66 \\
\hline & & Astrovirus & 5 & & 19.9 & 31.5 & - \\
\hline & & Sapovirus & 21 & & 25.6 & 28.3 & 0.50 \\
\hline
\end{tabular}

Cl, confidence interval; Cp, crossing point; Cq, quantification cycle; Ct, cycle threshold; GI/II, genogroup I/II; HR, hazard ratio; IQR, interquartile range; IV, intravenous; OR, odds ratio; PCR, polymerase chain reaction; ROC, receiver operating characteristic; rt, real-time; SD, standard deviation; UK, United Kingdom.

*Total number pathogen-specific positive samples in the study; number of PCR+ve samples used in the Ct analysis not provided.

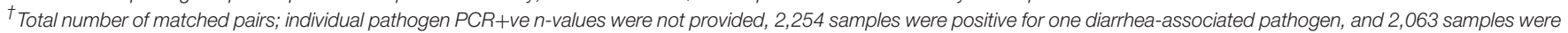
positive for $\geq 2$.

${ }^{\ddagger}$ Total number of PCR+ve patients in this study; number of patients included in the analysis of Ct value vs. symptom duration is unclear.

Bold indicates a statistically significant association.

Three studies investigated gastrointestinal viruses other than norovirus and rotavirus. In one study that investigated pathogen quantity and diarrhea in children $<5$ years old, associations between $\mathrm{Ct}$ value and diarrhea were reported for cases of adenovirus and astroviruses (29). No other associations between $\mathrm{Ct}$ values and cases vs. controls were identified $(27,30)$.

\section{Non-C. difficile Bacterial and Parasitic Pathogens}

Associations between patient clinical outcomes and the $\mathrm{Ct}$ value of non-C. difficile bacterial and parasitic pathogens were investigated in nine studies (Table 3).

Among bacterial studies, the majority investigated associations between quantitative PCR-derived bacterial loads and cases vs. controls (symptomatic vs. asymptomatic, or patients with vs. without diarrhea), and most studies found significant associations. Among five studies reporting differences in $\mathrm{Ct}$ values between cases vs. controls, significantly lower median Ct values were reported in cases of enterotoxigenic Escherichia coli (ETEC), enteropathogenic E. coli (EPEC), Campylobacter spp., enteroinvasive E. coli (EIEC)/Shigella spp., and Salmonella spp. (22, 27, 29, 30, 37). However, associations were not consistent across studies, including two reports $(n=9$ and $n=46)$ of no significant difference in cases vs. controls for Salmonella spp. $(27,30)$. In one study, associations were notably weaker for Campylobacter spp. and typical EPEC (29). In a study $(n=143)$ of patients with EPEC, a $29 \%$ increase in risk of diarrhea was observed for each $\log _{10}$ unit increase (calculated by Ct value) in bacterial load (OR 1.29; 95\% CI 1.08-1.53) (37).

Two studies also investigated associations between Ct values and bacterial disease severity. In cases of EIEC/Shigella spp., lower Ct values were significantly associated with higher vs. lower categories of disease severity $(n=286$; Ct value 25.3 vs. 36.6), dehydration ( $n=154$; OR $3.89 ; p=0.02)$, and requirement for intravenous fluids ( $n=154$; OR 2.29; $p=0.01)(27,38)$. Lower Ct values for ETEC-estA were significantly associated with vomiting ( $n=167$; OR $1.74 ; p=0.024)$ and with intravenous fluids ( $n=167$; OR $1.81 ; p=0.004)$, and Campylobacter spp. with vomiting ( $n=147$; OR $2.21 ; p=0.03)$ (27).

One study $(n=143)$ investigated the effect of EPEC bacterial load on the duration of symptoms; however, no significant association was observed (37).

All studies of parasites investigated associations between $\mathrm{Ct}$ values (or $\mathrm{Cq}$ ) and cases vs. controls (symptomatic vs. asymptomatic, or patients with vs. without diarrhea). In studies including Cryptosporidium spp., two reported significantly lower $\mathrm{Ct}$ values in cases vs. controls, including Elfving et al. $(n=67$; median Ct 32.1 vs. $36.8 ; p=0.0009)(22,30)$. One further study also reported lower $\mathrm{Ct}$ values in cases vs. controls $(n=23)$, but did not reach statistical significance (27). Furthermore, and contrary to expected results, Haque et al. reported higher mean $\mathrm{Ct}$ values in Cryptosporidium parvum and Cryptosporidium hominus cases than controls, although the differences were not significant ( $p=0.127$ and 0.098) (39). In a study in children $<5$ years $(n=\mathrm{N} / \mathrm{A})$, strong pathogen quantity-dependent associations 
TABLE 3 | Summary of studies that assessed PCR Ct values for non-C. difficile bacterial and parasitic pathogens against patient clinical presentation and outcomes.

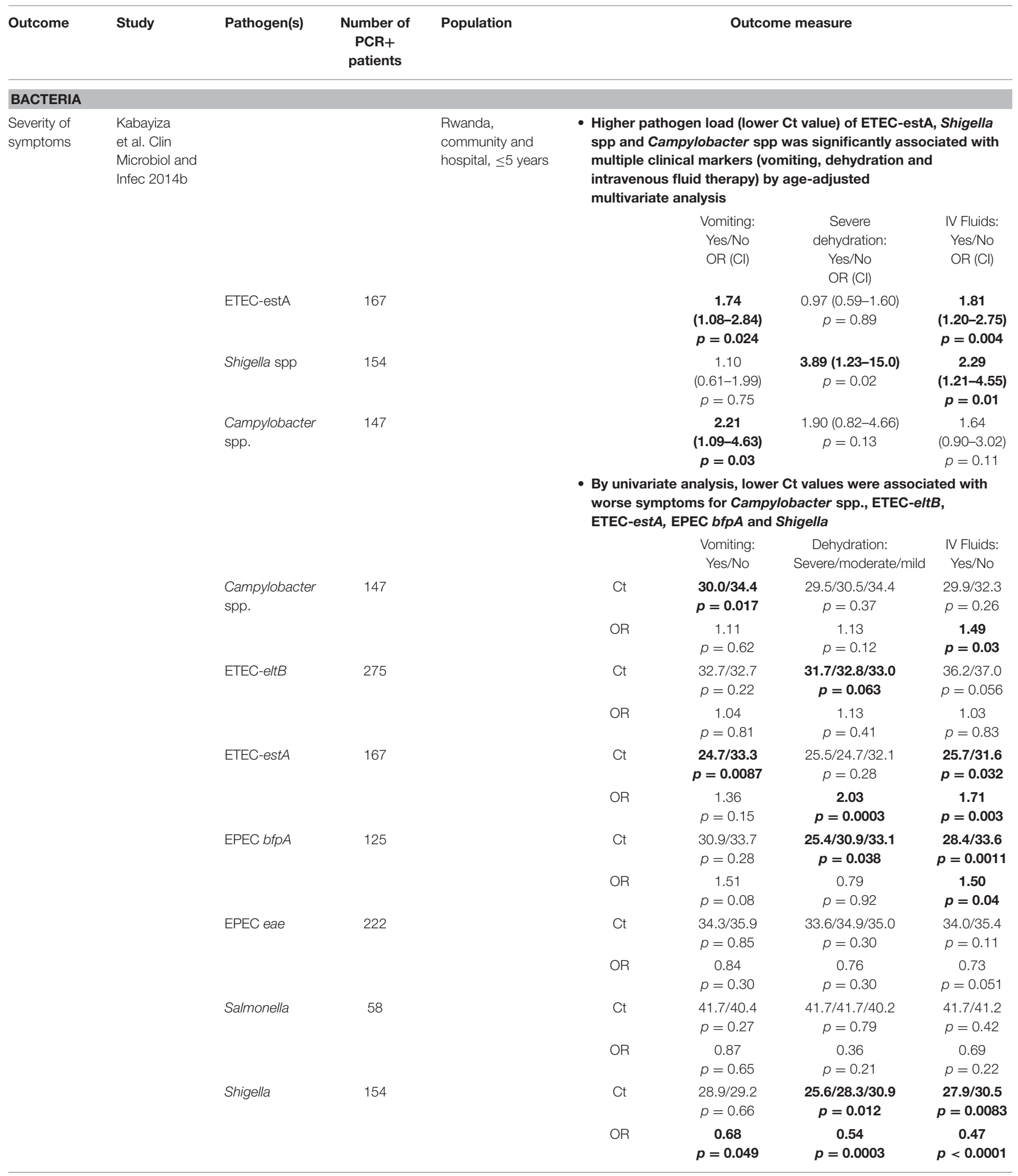


TABLE 3 | Continued

\begin{tabular}{|c|c|c|c|c|}
\hline Outcome & Study & Pathogen(s) & $\begin{array}{c}\text { Number of } \\
\text { PCR+ } \\
\text { patients }\end{array}$ & Population \\
\hline & $\begin{array}{l}\text { Vu DT et al. J } \\
\text { Clin Microbiol } \\
2004\end{array}$ & Shigella & 286 & $\begin{array}{l}\text { Vietnam, } \\
\text { community }\end{array}$ \\
\hline $\begin{array}{l}\text { Case s. } \\
\text { control }\end{array}$ & $\begin{array}{l}\text { Liu et al. } \\
\text { Lancet } 2016\end{array}$ & $\begin{array}{l}\text { Shigella spp., } \\
\text { EIEC, ETEC, } \\
\text { Campylobacter } \\
\text { jejuni or C } \\
\text { coli, EPEC, } \\
\text { Vibrio } \\
\text { cholerae, } \\
\text { Salmonella } \\
\text { spp, EAEC, } \\
\text { Aeromonas } \\
\text { spp }\end{array}$ & $5,304^{*}$ & $\begin{array}{l}\text { Bangladesh, India, } \\
\text { Pakistan, The } \\
\text { Gambia, Kenya, } \\
\text { Mali and } \\
\text { Mozambique, } \\
\text { community, <5 } \\
\text { years }\end{array}$ \\
\hline
\end{tabular}

- The trend between increasing number of rt-PCR cycles (Ct values) and decreasing disease severity was highly significant (p $<0.001)$

- The number of PCR cycles required to detect a PCR product was highest for patients $\geq 5$ years with culture-negative, non-bloody diarrheal specimens (36.6) and was lowest for children ( $<5$ years) with culture-positive, bloody diarrheal specimens $(25.3)(p<0.001)$

- Shigella spp. or EIEC, and heat-stable ETEC had strong quantity-dependent associations with diarrhea.

- For Shigella/EIEC, Cq values <33.1 were defined as "diarrhea-associated" and $<27.9$ were defined as "highly diarrhea-associated" (ROC cut-off 26.1; Youden index 0.18)

- For heat-stable ETEC, Cq values <26.2 were defined as "diarrhea-associated" and $<22.8$ were defined as "highly diarrhea-associated" (ROC cut-off 25.4; Youden index 0.25)

- Campylobacter jejuni or C coli and EPEC were only moderately associated with diarrhea

- For Campylobacter spp., Cq values < 19.7 were defined as "diarrhea-associated" and $<15.4$ were defined as "highly diarrhea-associated" (ROC cut-off 25.8; Youden index 0.08)

- For EPEC, Cq values < 19.5 were defined as "diarrhea-associated" and $<16.0$ were defined as "highly diarrhea-associated" (ROC cut-off 19.9; Youden index 0.07)

- Vibrio cholerae and Salmonella spp. showed associations with diarrhea

- For Vibrio cholerae, Cq values $<34.9$ were defined as "diarrhea-associated" and <33.8 were defined as "highly diarrhea-associated" (ROC cut-off 29.3; Youden index 0.55)

- For Salmonella spp, Cq values $<32.4$ were defined as "diarrhea-associated" and $<30.7$ were defined as "highly diarrhea-associated" (ROC cut-off 29.7; Youden index 0.29)

- EAEC and Aeromonas spp were associated with diarrhea only in specific study sites or age strata

$\begin{array}{llc}\begin{array}{ll}\text { Bruijnestein } \\ \text { et al. Clin }\end{array} & \text { Campylobacter } & 187 \\ \text { Microbiol } & \text { spp. } & \\ \text { Infect 2015 } & \text { Salmonella } & 32 \\ & \text { spp } & \\ & \text { E.coli } & 487 \\ & \text { ETEC } & 56 \\ & \text { Typical EPEC } & 20 \\ & \text { Atypical } & 227 \\ & \text { EPEC } & \\ & \text { STEC } & 37 \\ & \text { EAEC } & 127 \\ & \text { Shigella/EIEC } & 14\end{array}$

Elfving et al.

JCM 2014
187

32

487

56

20

27

37

14

4

- Significantly higher relative loads were observed for Campylobacter spp. ( $p<0.005)$, Salmonella spp. $(p<0.005)$, ETEC $(p<0.05)$ and typical EPEC $(p<0.005)$

- Ct values were significantly higher for STEC cases vs. controls

- No significant difference in Ct values between cases and controls $(p<0.05)$ were observed for EAEC or atypical EPEC

Netherlands primary Care
Zanzibar, community, 2 months -5 years

$\begin{array}{lc}\text { Campylobacter } & 112 \\ \text { ETEC-eltB } & 148 \\ \text { ETEC-estA } & 94\end{array}$

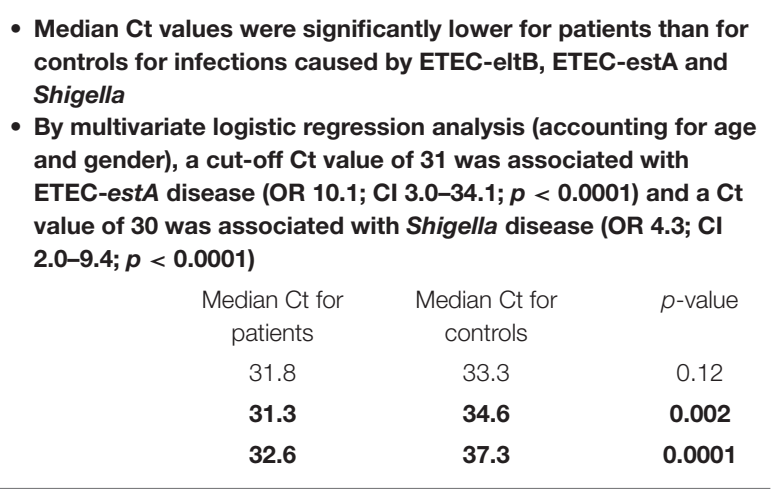

(Continued) 
TABLE 3 | Continued

\begin{tabular}{|c|c|c|c|c|c|c|c|c|}
\hline Outcome & Study & $\begin{array}{l}\text { Pathogen(s) } \\
\text { Salmonella }\end{array}$ & $\begin{array}{c}\begin{array}{c}\text { Number of } \\
\text { PCR+ } \\
\text { patients }\end{array} \\
13\end{array}$ & Population & \multicolumn{4}{|c|}{ Outcome measure } \\
\hline & & Shigella & 113 & & & 29.2 & 34.5 & $<0.0001$ \\
\hline & $\begin{array}{l}\text { Kabayiza } \\
\text { et al. Pediatr } \\
\text { Infect Dis J } \\
2014 \text { a }\end{array}$ & & & $\begin{array}{l}\text { Rwanda, } \\
\text { community and } \\
\text { hospital, } \leq 5 \text { years }\end{array}$ & $\begin{array}{r}\text { - Signific } \\
\text { Campy } \\
\text { bacteria }\end{array}$ & $\begin{array}{l}\text { ower Ct values } \\
\text { er, ETEC-estA } \\
\text { ions }\end{array}$ & $\begin{array}{l}\text { n patients vs. controls } \\
\text { ut not for other gastroin }\end{array}$ & $\begin{array}{l}\text { for } \\
\text { estinal }\end{array}$ \\
\hline & & & & & & $\begin{array}{l}\text { Median Ct for } \\
\text { patients }\end{array}$ & $\begin{array}{l}\text { Median Ct for } \\
\text { controls }\end{array}$ & $\begin{array}{l}p \text {-value } \\
(\mathrm{PCR}+)\end{array}$ \\
\hline & & Campylobacter & 121 & & & 29.75 & 33.02 & 0.007 \\
\hline & & ETEC-eltB & 213 & & & 33.91 & 34.15 & 0.90 \\
\hline & & ETEC-estA & 130 & & & 24.75 & 34.37 & 0.04 \\
\hline & $\begin{array}{l}\text { Barletta et al. } \\
\text { CID } 2011\end{array}$ & EPEC & 143 & $\begin{array}{l}\text { Peru, community, } \\
<2 \text { years }\end{array}$ & $\begin{array}{l}\text { - EPEC I } \\
\text { group l } \\
\text { - EPEC b } \\
\text { (144 vs. } \\
\text { - For a giv } \\
95 \% \mathrm{Cl} \\
\text { stool sar }\end{array}$ & $\begin{array}{l}\text { as significantly } \\
\text { 16) } \\
\text { load was simila } \\
\text { cteria/mg; } p=0 \\
\text { ld, the odds of c } \\
\text {.53) for each los }\end{array}$ & $\begin{array}{l}\text { igher in the diarrheal } \\
\text { Detween mild and moder } \\
\text { 22) } \\
\text { rrhea increased by } 29 \% \\
0 \text { unit increase in bacter }\end{array}$ & $\begin{array}{l}\text { s. control } \\
\text { ate cases } \\
\text { OR, 1.29; } \\
\text { load in the }\end{array}$ \\
\hline $\begin{array}{l}\text { Duration of } \\
\text { symptoms }\end{array}$ & $\begin{array}{l}\text { Barletta et al. } \\
\text { CID } 2011\end{array}$ & EPEC & 143 & $\begin{array}{l}\text { Peru, community, } \\
<2 \text { years }\end{array}$ & $\begin{array}{l}\text { - No differ } \\
\text { episode } \\
\text { days, re }\end{array}$ & $\begin{array}{l}\text { hacterial load } \\
\text { s. } 184 \text { vs. } 146 \text { b } \\
\text { ely) }\end{array}$ & $\begin{array}{l}\text { related to the duration } \\
\text { cteria/mg for } 7,7-14 \text {, an }\end{array}$ & $\begin{array}{l}\text { the } \\
d>14\end{array}$ \\
\hline \multicolumn{9}{|c|}{ PARASITES } \\
\hline & & & & & OR & $\begin{array}{c}0.90 \\
p=0.68\end{array}$ & $\begin{array}{c}0.51 \\
p=0.31\end{array}$ & $\begin{array}{c}1.68 \\
p=0.044\end{array}$ \\
\hline \multirow[t]{2}{*}{$\begin{array}{l}\text { Case vs. } \\
\text { control }\end{array}$} & $\begin{array}{l}\text { Liu et al. } \\
\text { Lancet } 2016\end{array}$ & $\begin{array}{l}\text { Cryptosporidium } \\
\text { spp., } \\
\text { Cyclospora } \\
\text { cayetanensis, } \\
\text { Entamoeba } \\
\text { histolytica. }\end{array}$ & $5,304^{*}$ & $\begin{array}{l}\text { Bangladesh, India, } \\
\text { Pakistan, The } \\
\text { Gambia, Kenya, } \\
\text { Mali and } \\
\text { Mozambique, } \\
\text { community, <5 } \\
\text { years }\end{array}$ & \multicolumn{4}{|c|}{$\begin{array}{l}\text { - Cryptosporidium spp, had strong quantity-dependent } \\
\text { associations with diarrhea } \\
\text { - Cq values }<29.1 \text { were defined as "diarrhea-associated" and }<24.0 \\
\text { were defined as "highly diarrhea-associated" (ROC cut-off } 27.5 \text {; } \\
\text { Youden index 0.17) } \\
\text { - Cyclospora cayetanensis and Entamoeba histolytica showed } \\
\text { associations with diarrhea } \\
\text { - For Cyclospora cayetanensis, Cq values }<29.6 \text { were defined as } \\
\text { "highly diarrhea-associated" (ROC cut-off } 34.0 \text {; Youden index 0.40) } \\
\text { - For Entamoeba histolytic, Cq values }<34.8 \text { were defined as } \\
\text { "diarrhea-associated" and <32.6 were defined as "highly } \\
\text { diarrhea-associated" (ROC cut-off 26.9; Youden index 0.48) }\end{array}$} \\
\hline & $\begin{array}{l}\text { Bruijnestein } \\
\text { et al. Clin } \\
\text { Microbiol } \\
\text { Infect } 2015\end{array}$ & $\begin{array}{l}\text { C.parvum/ } \\
\text { hominis } \\
\text { Giardia } \\
\text { lamblia }\end{array}$ & $\begin{array}{l}56 \\
118\end{array}$ & $\begin{array}{l}\text { Netherlands, } \\
\text { primary Care }\end{array}$ & \multicolumn{4}{|c|}{$\begin{array}{l}\text { - Significantly higher relative loads were observed for } \mathbf{C} \text {. } \\
\text { parvum/hominis }(\boldsymbol{p}<\mathbf{0 . 0 5 )} \\
\text { - Higher loads were observed for G. lamblia, although statistical } \\
\text { significance was not reached; } p=0.084\end{array}$} \\
\hline
\end{tabular}


TABLE 3 | Continued

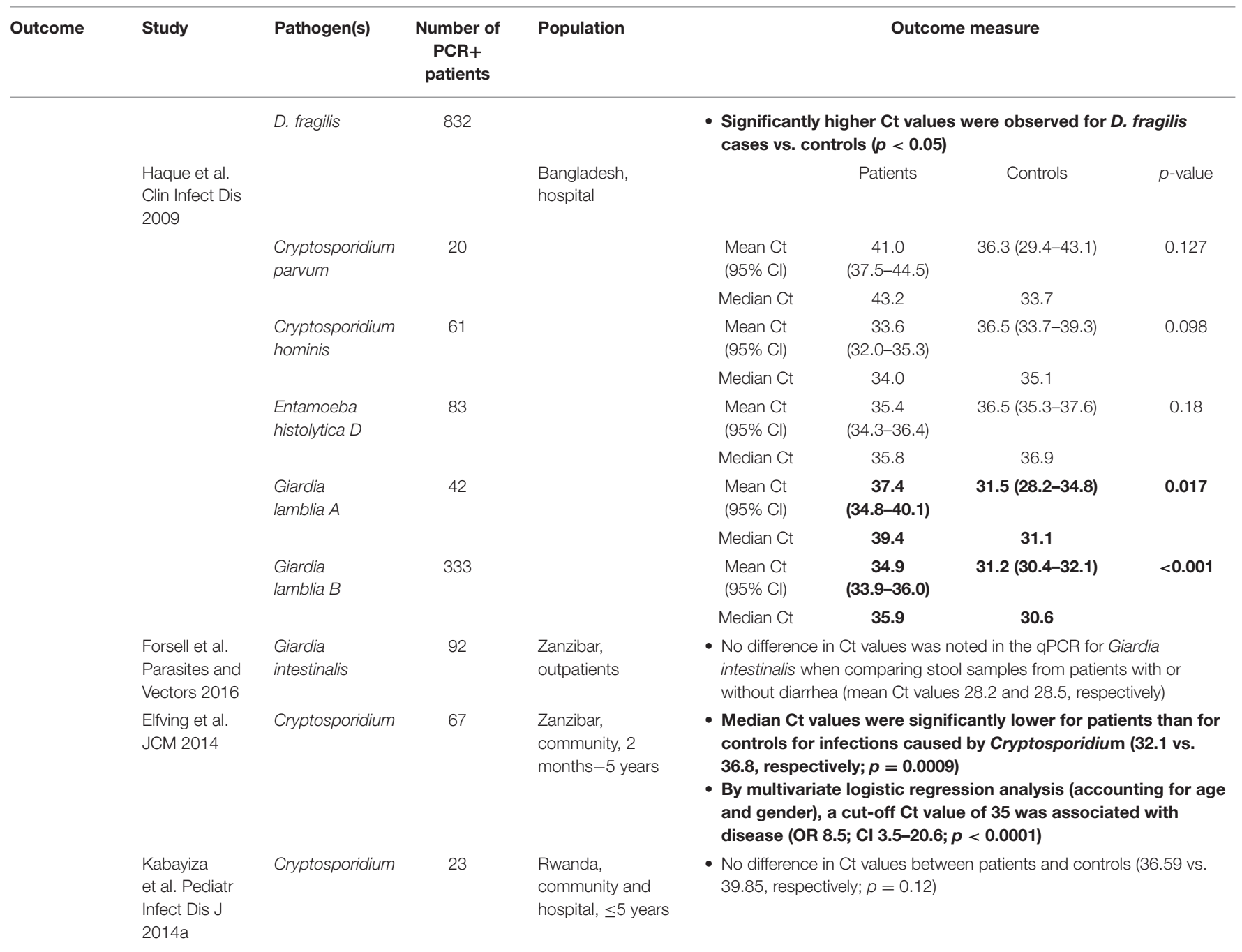

Cl, confidence interval; $C p$, crossing point; Cq, quantification cycle; Ct, cycle threshold; EAEC, enteroaggregative E. coli; EIEC, enteroinvasive E. coli; EPEC, enteropathogenic E. coli; ETEC, enterotoxigenic E. coli; IV, intravenous; OR, odds ratio; PCR, polymerase chain reaction; qPCR, quantitative polymerase chain reaction; ROC, receiver operating characteristic; STEC, Shiga toxin-producing E. coll; UK, United Kingdom.

${ }^{\star} T$ Total number of matched pairs; individual pathogen PCR+ve $n$ values were not provided, 2,254 samples were positive for one diarrhea-associated pathogen, and 2,063 samples were positive for $\geq 2$.

Bold indicates a statistically significant association.

with diarrhea were reported in cases of Cryptosporidium spp. (29). Among three studies including Giardia spp. ( $n=118$, $n=375$ and $n=92$ ), none reported statistically significant lower Ct values in cases vs. controls (22, 39, 40). Notably, Haque et al. reported that Giardia lamblia parasite load, as measured by Ct values, was inversely related to diarrhea, which the authors suggest could be related to the primary role played by the immune system in diarrheal illness that results from these infections (39).

\section{DISCUSSION}

The objective of this systematic review was to assess the global medical literature for any correlation between $\mathrm{Ct}$ values and clincal outcomes of patients with gastrointestinal infections. Lower $\mathrm{Ct}$ values correspond with greater quantities of detectible target gene and therefore a higher pathogen load, which may correspond with less favorable clinical outcomes. Here we report outcomes from studies identified that report on gastrointestinal pathogens only. This review gathers data from 33 studies, with the largest number of studies for $C$. difficile $(n=15)$. The most common outcomes reported were related to symptoms, including case vs. control, with vs. without diarrhea, and severity of symptoms.

Evidence in this review suggests associations between $\mathrm{Ct}$ values and symptomatic $C$. difficile infections. Four out of eight studies reporting the association between lower $\mathrm{Ct}$ values and increased disease severity found the association to be significant, 
including two studies that reported lower $\mathrm{Ct}$ values as a predictor of poor outcome $(14,17)$. Furthermore, $2 / 4$ case vs. control studies reported significantly lower Ct values in symtomatic cases. Most of the $C$. difficile studies reported genes encoding toxin $\mathrm{A} / \mathrm{B}$ as the target for PCR diagnostics, which when detected by other methods, is generally inferred as marker of disease severity (5).

All studies of norovirus and rotavirus reported lower $\mathrm{Ct}$ values in cases vs. controls; the majority for norovirus GII and $\sim 50 \%$ for rotavirus reported significant differences. Furthermore, two studies of rotavirus infections reported significant associatons between lower $\mathrm{Ct}$ values and severity of symptoms, including vomiting, severe dehydration and administering intravenous fluids $(27,34)$. Notably, the association of $\mathrm{Ct}$ values and symptom severity was more pronounced for norovirus GII than norovirus GI $(25,27,28)$. One possible explanation for this is the increased virulence observed with GII infection compared with other norovirus genogroups (41), although more investigation is necessary to draw firm conclusions.

This review found less evidence for the clinical utility of $\mathrm{Ct}$ values in non-C. difficile bacterial and parasitic infections compared with C. difficile and gastrointestinal viruses. Multiple studies reported significant associations between bacterial loads and symptomatic cases, particularly for Shigella $(29,30)$. Two studies reported Shigella association with symptom severity $(27,38)$. Inconsistencies were found in studies of parasitic infections; some studies indicated an association between low Ct values and symptomatic infection in patients with Cryptosporidium spp., however, evidence is limited (22, 29, 30). There is insufficient evidence to draw conclusions for other parasitic infections.

Among the studies included in this review, evidence suggests that $\mathrm{Ct}$ values may have utility in defining symptomatic causality, particularly in cases of polymicrobial infection. In one study of norovirus-positive samples, coinfection with rotavirus was observed in 3.7 and $7.4 \%$ of asymptomatic and diarrheal samples, respectively; probable etiology was determined based on relative $\mathrm{Ct}$ values, highlighting their utility for defining causitive organisms in this setting (28). Ct values may also aid causative diagnosis in patients with $C$. difficille infection, where asymptomatic colonization $(5,6)$, and coinfections have been reported (42). C. difficile fecal load is already considered to be of diagnostic utility in distinguishing between infection and colonization $(43,44)$. However, it is essential to consider Ct values within the context of clinical presentation rather than utilize $\mathrm{Ct}$ values as an independent marker of disease.

Despite multiple studies reporting significant associations between high genomic load (low Ct values) and symptomatic infections, particularly for $C$. difficile, norovirus and rotavirus, statistically significant evidence was inconsistent across studies despite similar trends. A possible explanation for this is the diversity of populations investigated across each study (e.g., hospital vs. community setting, pediatric vs. adult populations); adjusting for similar settings may uncover stronger trends toward $\mathrm{Ct}$ value and patient outcomes. Further assessments of associations between $\mathrm{Ct}$ values and
LOS, hospital/ICU admission, for example, could also aid in understanding the utility of $\mathrm{Ct}$ values in the diagnosis of gastrointestinal infections.

When interpreting the studies in this systematic review, consideration must be given to the settings and populations in which they were conducted. Studies for some pathogens, such as norovirus, were conducted primarily in pediatric populations and as such their conclusions may not apply to adult populations. All but one of the studies investigating non-C. difficile bacterial pathogens and parasites were performed in non-industrialized countries; therefore, the clinical impact of $\mathrm{Ct}$ values for these pathogens in industrialized countries remains to be determined. Of the seven studies that detected parasites, five investigated a large list of GI pathogens and multiple pathogens were detected for $8-72 \%$ patients $(22,27,29,30)$. These studies highlight the utility of syndromic testing in gastrointestinal infection, where multiplex testing is able to detect more pathogens and coinfections than conventional methods (42). It should also be noted that multiplex PCR for GI pathogens does not currently provide a picture of the microbiome, whereas culture-based techniques are able to provide an understanding of dysbiosis resulting from GI infections.

Differences in study methodology and qPCR workflow are likely to impact $\mathrm{Ct}$ values, including: specimen source, collection method, transport media type and volume, stability, quality of the sample, time of sampling vs. onset of infection, master mix components, type and concentration of passive reference dye, reaction efficiency, inter- and intravariability in assay platforms, and whether they were single or multiplex systems. Methodologies varied widely between studies and many (39\%) had some or many gaps in reporting defined standardized methodologies. Therefore, within-study variability may have limited the ability to detect associations. The majority (84.8\%; 28/33) of studies did not report normalized Ct values, which would have provided more accurate estimations of genomic load for each sample. Although outside the scope of our review, we noted not all $(66.7 \%$; $22 / 33)$ studies presented Ct value distributions. Further studies to understand the distribution of Ct values in relation to patient outcomes across the populations would be necessary if $\mathrm{Ct}$ values are to be utilized in clinical decision-making. After data analysis had been completed, we became aware of the Minimum Information for Publication of Quantitative rt-PCR Experiments (MIQE) guidelines (45), which should be applied to laboratory-developed tests. Some of the studies utilized in this review use commercially available assays and, therefore, when implemented in clinical diagnostic routines, applicable validation, and verification using external controls are necessary. Due to the late discovery it was not possible to re-assess the studies using laboratory-developed assays with the MIQE guidelines in mind; however, we believe that assessment of study methodology using these guidelines would not significantly alter the findings of this systematic review.

There were a number of limitations to this systematic review. The protocol restricted articles referring to $\mathrm{Ct}$ values as a measure of genomic load, therefore studies which reported genomic load in measures other than Ct value were not picked up in the database searches or excluded from during 
screening. Furthermore, articles describing Ct values but with no mention of $\mathrm{Ct}$ values in the title, abstract or keywords, were not retrieved based on the search parameters used in the database searches. In addition, late in the review we became aware of alternative wording for $\mathrm{Ct}$ values, including $\mathrm{Cq}$ and "crossing point" [discussed in detail in (45)]; while we have added articles with these terms manually, it is possible that some may have been missed. Another limitation to this review was the assessment of all included studies as poor quality for bias by the Newcastle-Ottawa scale. This is due to the majority of studies reporting $\mathrm{Ct}$ values as secondary outputs, as opposed to seeking to compare clinical outcomes against $\mathrm{Ct}$ values. Consequently, the studies did not fully align with the risk and bias assessment. There was considerable variability between studies. Given the high heterogeneity between studies, it was not possible to conduct aggregated/meta-analyses, a key limitation in the scope of this review. A number of studies only made comparative analysis between symptomatic and asymptomatic cases, which limits the clinical utility of these studies in defining $\mathrm{Ct}$ values as a measure of disease severity. However, Ct values of asymptomatic patients still hold clinical value in order to discriminate between infection and colonization, an observation reported in multiple studies (4$6,25,27)$. A single reviewer conducted the data extraction and a second reviewer checked all the data points. Whilst an acceptable approach, the methodology could have been optimized by double independent reviewer data extraction with a third reviewer for discrepancy resolution. Due to the large number of studies identified as potential data sources for this review, the single-reviewer extraction method ensured that the review remained feasible. Despite these limitations, we believe this review provides insights into the potential clinical utility of gastrointestinal pathogen $\mathrm{Ct}$ values. In summary, there is evidence to support relationships between $\mathrm{Ct}$ values and clinical outcomes in gastrointestinal infections. Considered alongside clinical presentation, Ct values could help to guide treatment decisions, particularly in cases of $C$. difficile, where treatment is guided by severity of disease and asymptomatic colonization

\section{REFERENCES}

1. GBD 2017 Causes of Death Collaborators. Global, regional, and national agesex-specific mortality for 282 causes of death in 195 countries and territories, 1980-2017: a systematic analysis for the Global Burden of Disease Study 2017. Lancet. (2018) 392:1736-88. doi: 10.1016/S0140-6736(18)32203-7

2. GBD 2017 Diarrhoeal Disease Collaborators. Quantifying risks and interventions that have affected the burden of diarrhoea among children younger than 5 years: an analysis of the Global Burden of Disease Study 2017. Lancet Infect Dis. (2020) 20:37-59. doi: 10.1016/S1473-3099(19)30401-3

3. GBD 2016 Diarrhoeal Disease Collaborators. Estimates of the global, regional, and national morbidity, mortality, and aetiologies of diarrhoea in 195 countries: a systematic analysis for the Global Burden of Disease Study 2016. Lancet Infect Dis. (2018) 18:1211-28. doi: 10.1016/S1473-3099(18)30362-1

4. Phillips G, Lopman B, Tam CC, Iturriza-Gomara M, Brown D, Gray J. Diagnosing norovirus-associated infectious intestinal disease using viral load. BMC Infect Dis. (2009) 9:63. doi: 10.1186/1471-2334-9-63

5. Crobach MJT, Duszenko N, Terveer EM, Verduin CM, Kuijper EJ. Nucleic acid amplification test quantitation as predictor of toxin has been observed $(5,6,46)$. This review did not uncover sufficient evidence to draw conclusions on the clinical utility of $\mathrm{Ct}$ values for non-C. difficile bacterial and parasitic infections. This systematic review is the first to assess the relationship between $\mathrm{Ct}$ values and clinical outcomes in gastrointestinal infections, large-scale clinical trials with endpoints centered on Ct values are warranted to draw definitive evidence.

\section{DATA AVAILABILITY STATEMENT}

The original contributions presented in the study are included in the article/Supplementary Material, further inquiries can be directed to the corresponding author/s.

\section{AUTHOR CONTRIBUTIONS}

$\mathrm{BV}, \mathrm{DB}, \mathrm{JP}, \mathrm{SR}, \mathrm{DM}, \mathrm{GH}$, and JV were involved in conception and design of the study. All authors contributed to interpretation of the data, manuscript drafting and revision, and approved the submitted version.

\section{FUNDING}

Medical writing support for the development of this manuscript, under the direction of the authors, was provided by Isabella Talbot, BSc, of Ashfield MedComms, an Ashfield Health company, and funded by Qiagen Manchester Ltd. This study received funding from QIAGEN Manchester Ltd. The funder had the following involvement with the study: article processing fees and provision of medical writing support.

\section{SUPPLEMENTARY MATERIAL}

The Supplementary Material for this article can be found online at: https://www.frontiersin.org/articles/10.3389/fmed. 2021.711809/full\#supplementary-material

presence in Clostridium difficile infection. J Clin Microbiol. (2018) 56:17. doi: 10.1128/JCM.01316-17

6. Hecht S, Wang H, Everhart K, Watson J, Leber A. Understanding the clinical implications of Clostridium difficile detection in the molecular age: colonization vs infection in children less than 3 years of age. Open Forum Infect Dis. (2019) 6:S812. doi: 10.1093/ofid/ofz360.2036

7. Pouletty M, De Pontual L, Lopez M, Morin L, Poilane I, Pham LL, et al. Multiplex PCR reveals a high prevalence of multiple pathogens in traveller's diarrhoea in children. Arch Dis Child. (2019) 104:1416. doi: 10.1136/archdischild-2017-314327

8. Boers SA, Peters CJA, Wessels E, Melchers WJG, Claas ECJ. Performance of the QIAstat-Dx gastrointestinal panel for diagnosing infectious gastroenteritis. J Clin Microbiol. (2020) 58:e01737-19. doi: 10.1128/JCM.01737-19

9. Rao SN, Manissero D, Steele VR, Pareja J. A systematic review of the clinical utility of cycle threshold values in the context of COVID-19. Infect Dis Ther. (2020) 9:573-86. doi: 10.1007/s40121-020-00324-3

10. Rabaan AA, Tirupathi R, Sule AA, Aldali J, Mutair AA, Alhumaid S, et al. Viral dynamics and real-time RT-PCR Ct values correlation with disease severity 
in COVID-19. Diagnostics. (2021) 11:61091. doi: 10.3390/diagnostics11 061091

11. Wells GA, Shea B, O'Connell D, Peterson J, Welch V, Losos M, Tugwell P. The Newcastle Ottawa Scale (NOS) for Assessing the Quality of Nonrandomised Studies in Meta-Analyses. Available online at: http://www.ohri.ca/programs/ clinical_epidemiology/oxford (accessed September 03, 2021).

12. Rao K, Micic D, Natarajan M, Winters S, Kiel MJ, Walk ST, et al. Clostridium difficile ribotype 027: relationship to age, detectability of toxins $\mathrm{A}$ or $\mathrm{B}$ in stool with rapid testing, severe infection, and mortality. Clin Infect Dis. (2015) 61:233-41. doi: 10.1093/cid/civ254

13. Davies KA, Planche T, Wilcox MH. The predictive value of quantitative nucleic acid amplification detection of Clostridium difficile toxin gene for faecal sample toxin status and patient outcome. PLOS ONE. (2018) 13:e0205941. doi: 10.1371/journal.pone.0205941

14. Jazmati N, Hellmich M, Licanin B, Plum G, Kaasch AJ. PCR cycle threshold value predicts the course of Clostridium difficile infection. Clin Microbiol Infect. (2016) 22:e7-8. doi: 10.1016/j.cmi.2015.09.012

15. Kamboj M, Brite J, Mcmillen T, Robilotti E, Herrera A, Sepkowitz K, et al. Potential of real-time PCR threshold cycle (CT) to predict presence of free toxin and clinically relevant $C$. difficile infection (CDI) in patients with cancer. J Infect. (2018) 76:369-75. doi: 10.1016/j.jinf.2017.12.001

16. De Francesco MA, Lorenzin G, Piccinelli G, Corbellini S, Bonfanti C, Caruso A. Correlation between tcdB gene PCR cycle threshold and severe Clostridium difficile disease. Anaerobe. (2019) 59:141-4. doi: 10.1016/j.anaerobe.2019.06.017

17. Reigadas E, Alcala L, Valerio M, Marin M, Martin A, Bouza E. Toxin B PCR cycle threshold as a predictor of poor outcome of Clostridium difficile infection: a derivation and validation cohort study. J Antimicrob Chemother. (2016) 71:1380-5. doi: 10.1093/jac/dkv497

18. Anikst VE, Gaur RL, Schroeder LF, Banaei N. Organism burden, toxin concentration, and lactoferrin concentration do not distinguish between clinically significant and nonsignificant diarrhea in patients with Clostridium difficile. Diagn Microbiol Infect Dis. (2016) 84:3436. doi: 10.1016/j.diagmicrobio.2015.11.022

19. Sante L, Pedroso Y, Castro B, Lecuona M. Is there a relationship between the polimerase chain reaction cycle threshold and the risk of severe Clostridium difficile infection? Enfermedades Infecciosas Microbiologia Clinica. (2018) 36:600-1. doi: 10.1016/j.eimce.2017.11.022

20. Origuen J, Orellana MA, Fernandez-Ruiz M, Corbella L, San Juan R, Ruiz-Ruigomez M, et al. Toxin B PCR amplification cycle threshold adds little to clinical variables for predicting outcomes in Clostridium difficile infection: a retrospective cohort study. J Clin Microbiol. (2019) 57:e01125-18. doi: 10.1128/JCM.01125-18

21. Bub F, Chatigre JK, Yao JA, Coulibaly JT, Von Mueller L, Polman $\mathrm{K}$, et al. Polymerase chain reaction for the diagnosis of Clostridium difficile infection in patients with persistent digestive disorders and asymptomatic controls in Cote d'Ivoire. Trop Med Internat Health. (2017) 22:3S7.1. doi: 10.1097/MPG.0b013e3182999990

22. Bruijnesteijn Van Coppenraet LES, Dullaert-De Boer M, Ruijs GJHM, Van Der Reijden WA, Van Der Zanden AGM, Weel JFL, et al. Case-control comparison of bacterial and protozoan microorganisms associated with gastroenteritis: application of molecular detection. Clin Microbiol Infect. (2015) 21:592.e599-19. doi: 10.1016/j.cmi.2015.02.007

23. El Feghaly RE, Stauber JL, Deych E, Gonzalez C, Tarr PI, Haslam DB. Markers of intestinal inflammation, not bacterial burden, correlate with clinical outcomes in Clostridium difficile infection. Clin Infect Dis. (2013) 56:1713-21. doi: 10.1093/cid/cit147

24. El Feghaly RE, Stauber JL, Tarr PI, Haslam DB. Intestinal inflammatory biomarkers and outcome in pediatric Clostridium difficile infections. J Pediatr. (2013) 163:1697-704.e1692. doi: 10.1016/j.jpeds.2013.07.029

25. Kabue JP, Meader E, Hunter PR, Potgieter N. Norovirus prevalence and estimated viral load in symptomatic and asymptomatic children from rural communities of Vhembe district, South Africa. J Clin Virol. (2016) 84:128. doi: 10.1016/j.jcv.2016.09.005

26. Dung TT, Phat VV, Nga TV, My PV, Duy PT, Campbell JI, et al. The validation and utility of a quantitative one-step multiplex RT real-time PCR targeting rotavirus A and norovirus. J Virol Methods. (2013) 187:13843. doi: 10.1016/j.jviromet.2012.09.021
27. Kabayiza JC, Andersson ME, Nilsson S, Bergstrom T, Muhirwa G, Lindh M. Real-time PCR identification of agents causing diarrhea in Rwandan children less than 5 years of age. Pediatr Infect Dis J. (2014) 33:103742. doi: 10.1097/INF.0000000000000448

28. Saito M, Goel-Apaza S, Espetia S, Velasquez D, Cabrera L, Loli S, et al. Multiple norovirus infections in a birth cohort in a Peruvian Periurban community. Clin Infect Dis. (2014) 58:483-91. doi: 10.1093/cid/cit763

29. Liu J, Platts-Mills JA, Juma J, Kabir F, Nkeze J, Okoi C, et al. Use of quantitative molecular diagnostic methods to identify causes of diarrhoea in children: a reanalysis of the GEMS case-control study. Lancet. (2016) 388:1291-301. doi: 10.1016/S0140-6736(16)31529-X

30. Elfving K, Andersson M, Msellem MI, Welinder-Olsson C, Petzold M, Bjorkman A, et al. Real-time PCR threshold cycle cutoffs help to identify agents causing acute childhood diarrhea in Zanzibar. J Clin Microbiol. (2014) 52:916-23. doi: 10.1128/JCM.02697-13

31. Gustavsson L, Westin J, Lindh M, Andersson LM. Faecal viral load does not predict short-term mortality in norovirus infection. J Clin Virol. (2015) 70:S57. doi: 10.1016/j.jcv.2015.07.135

32. Partridge DG, Evans CM, Raza M, Kudesia G, Parsons HK. Lessons from a large norovirus outbreak: impact of viral load, patient age and ward design on duration of symptoms and shedding and likelihood of transmission. J Hosp Infect. (2012) 81:25-30. doi: 10.1016/j.jhin.2012.02.002

33. Phillips G, Lopman B, Tam CC, Iturriza-Gomara M, Brown D, Gray J. Diagnosing rotavirus A associated IID: Using ELISA to identify a cut-off for real time RT-PCR. J Clin Virol. (2009) 44:242-5. doi: 10.1016/j.jcv.2008.12.001

34. Kang G, Iturriza-Gomara M, Wheeler JG, Crystal P, Monica B, Ramani S, et al. Quantitation of group A rotavirus by real-time reverse-transcriptionpolymerase chain reaction: correlation with clinical severity in children in South India. J Med Virol. (2004) 73:118-22. doi: 10.1002/jmv.20053

35. Ramani S, Sankaran P, Arumugam R, Sarkar R, Banerjee I, Mohanty I, et al. Comparison of viral load and duration of virus shedding in symptomatic and asymptomatic neonatal rotavirus infections. J Med Virol. (2010) 82:18037. doi: $10.1002 /$ jmv. 21872

36. Mukhopadhya I, Sarkar R, Menon VK, Babji S, Paul A, Rajendran P, et al. Rotavirus shedding in symptomatic and asymptomatic children using reverse transcription-quantitative PCR. J Med Virol. (2013) 85:16618. doi: $10.1002 /$ jmv.23641

37. Barletta F, Ochoa TJ, Mercado E, Ruiz J, Ecker L, Lopez G, et al. Quantitative real-time polymerase chain reaction for enteropathogenic Escherichia coli: a tool for investigation of asymptomatic versus symptomatic infections. Clin Infect Dis. (2011) 53:1223-9. doi: 10.1093/cid/cir730

38. Vu DT, Sethabutr O, Von Seidlein L, Tran VT, Do GC, Bui TC, et al. Detection of Shigella by a PCR assay targeting the ipaH gene suggests increased prevalence of shigellosis in Nha Trang, Vietnam. J Clin Microbiol. (2004) 42:2031-5. doi: 10.1128/JCM.42.5.2031-2035.2004

39. Haque R, Mondal D, Karim A, Molla IH, Rahim A, Faruque AS, et al. Prospective case-control study of the association between common enteric protozoal parasites and diarrhea in Bangladesh. Clin Infect Dis. (2009) 48:1191-7. doi: 10.1086/597580

40. Forsell J, Granlund M, Samuelsson L, Koskiniemi S, Edebro H, Evengard B. High occurrence of Blastocystis sp. subtypes 1-3 and Giardia intestinalis assemblage B among patients in Zanzibar. Tanzania Parasit Vectors. (2016) 9:370. doi: 10.1186/s13071-016-1637-8

41. Huhti L, Szakal ED, Puustinen L, Salminen M, Huhtala H, Valve O, et al Norovirus GII-4 causes a more severe gastroenteritis than other noroviruses in young children. J Infect Dis. (2011) 203:1442-4. doi: 10.1093/infdis/jir039

42. Stockmann C, Rogatcheva M, Harrel B, Vaughn M, Crisp R, Poritz M, et al. How well does physician selection of microbiologic tests identify Clostridium difficile and other pathogens in paediatric diarrhoea? Insights using multiplex PCR-based detection. Clin Microbiol Infect. (2015) 21:179.e17915. doi: 10.1016/j.cmi.2014.07.011

43. Leslie JL, Cohen SH, Solnick JV, Polage CR. Role of fecal Clostridium difficile load in discrepancies between toxin tests and PCR: is quantitation the next step in C. difficile testing? Eur J Clin Microb Infect Dis. (2012) 31:32959. doi: 10.1007/s10096-012-1695-6

44. Dionne L-L, Raymond F, Corbeil J, Longtin J, Gervais P, Longtin Y. Correlation between Clostridium difficile bacterial load, commercial realtime PCR cycle thresholds, and results of diagnostic tests based on enzyme 
immunoassay and cell culture cytotoxicity assay. J Clin Microbiol. (2013) 51:3624-30. doi: 10.1128/JCM.01444-13

45. Bustin SA, Benes V, Garson JA, Hellemans J, Huggett J, Kubista M, et al. The MIQE guidelines: minimum information for publication of quantitative real-time PCR experiments. Clin Chem. (2009) 55:61122. doi: 10.1373/clinchem.2008.112797

46. Public Health England. Updated Guidance on the Management and Treatment of Clostridium difficile Infection. (2013). Available online at: https://www. gov.uk/government/publications/clostridium-difficile-infection-guidanceonmanagement-and-treatment (accessed March 31, 2021).

Conflict of Interest: JP, SR, and DM are employed by Qiagen. BV reports grants, personal fees and non-financial support from Qiagen, personal fees and non-financial support from BioMérieux, personal fees from Hologic, personal fees from Gilead, outside the submitted work. DB reports personal fees from Qiagen, outside the submitted work.

The remaining authors declare that the research was conducted in the absence of any commercial or financial relationships that could be construed as a potential conflict of interest.
The authors declare that this study received funding from Qiagen Manchester Ltd. The funder had the following involvement in the study: article processing fees and provision of medical writing support. The funder was not involved in the study design, collection, analysis, interpretation of data, the writing of this article or the decision to submit it for publication.

Publisher's Note: All claims expressed in this article are solely those of the authors and do not necessarily represent those of their affiliated organizations, or those of the publisher, the editors and the reviewers. Any product that may be evaluated in this article, or claim that may be made by its manufacturer, is not guaranteed or endorsed by the publisher.

Copyright (C) 2021 Bonacorsi, Visseaux, Bouzid, Pareja, Rao, Manissero, Hansen and Vila. This is an open-access article distributed under the terms of the Creative Commons Attribution License (CC BY). The use, distribution or reproduction in other forums is permitted, provided the original author(s) and the copyright owner(s) are credited and that the original publication in this journal is cited, in accordance with accepted academic practice. No use, distribution or reproduction is permitted which does not comply with these terms. 